Y. Billaud, D. Saury \& D. Lemonnier (2017) Numerical investigation of coupled natural convection and radiation in a differentially heated cubic cavity filled with humid air. Effects of the cavity size, Numerical Heat Transfer, Part A: Applications, 72:7, 495-518, DOI: 10.1080/10407782.2017.1386509

https://doi.org/10.1080/10407782.2017.1386509

\title{
Numerical investigation of coupled natural convection and radiation in a differentially heated cubic cavity filled with humid air. Effects of the cavity size
}

\author{
Y. Billaud ${ }^{\mathrm{a}, *}$, D. Saury ${ }^{\mathrm{a}}$, D. Lemonnier ${ }^{\mathrm{a}}$ \\ ${ }^{a}$ Institut Pprime UPR CNRS 3346 - CNRS / ENSMA / Univ. Poitiers, 1 avenue Clement Ader, B.P. 40109, \\ F-86961 Futuroscope Chasseneuil CEDEX, FRANCE
}

\begin{abstract}
A numerical study of natural convection with surface and air $/ \mathrm{H}_{2} \mathrm{O}$ mixture radiation in a differentially heated cubic square cavity is presented. The coupled flow and heat transfers in the cavity are predicted by coupling a finite volume method with a spectral line weighted sum of gray gases model (SLW) to describe gas radiative properties. The radiative transfer equation (RTE) is solved by means of the discrete ordinates method (DOM). Simulations are performed at $R a=10^{6}$, considering different combinations of passive wall and/or gas radiation properties and different cavity length. It was found that in presence of a participative medium representative of building, cavity length has a strong influence on temperature and velocity fields which affect the global circulation and the heat transfers in the cavity. For each steady state solution, the convective and radiative contributions to the global heat transfer are discussed. More specifically, boundary layers thickness, thermal stratification parameter and three-dimensional effects are compared to pure convective case results. The results suggest that radiative effects, often considered as negligible in view of the relatively low optical thickness, may not be neglected when trying to predict regime transitions.
\end{abstract}

\section{Nomenclature}

$\begin{array}{ll}a_{j} & \text { weighting coefficient associated with the } j^{\text {th }} \text { fictitious gray gas } \\ C_{a b s} & \text { absorption cross section }\left(\mathrm{m}^{2} / \mathrm{mol}\right) \\ C_{p} & \text { heat capacity }(\mathrm{J} / \mathrm{kg} \cdot \mathrm{K}) \\ F & \text { black body distribution function } \\ \vec{g} & \text { gravitational field }\left(\mathrm{m} / \mathrm{s}^{2}\right) \\ G_{\lambda} & \text { spectral incident radiation }\left(\mathrm{W} / \mathrm{m}^{2}\right) \\ I_{\lambda} & \text { spectral intensity }\left(\mathrm{W} / \mathrm{m}^{2} . \mathrm{sr}\right) \\ I_{b \lambda} & \text { spectral black body intensity }\left(\mathrm{W} / \mathrm{m}^{2} . s r\right) \\ L & \text { cubic cavity length }(\mathrm{m}) \\ N_{g} & \text { total number of fictitious gases } \\ q_{r}^{i n c} & \text { incident radiative heat flux }\left(\mathrm{W} \cdot \mathrm{m}^{2}\right) \\ R & \text { ideal gas constant, } R=8.3144621(\mathrm{~J} / \mathrm{K} . \mathrm{mol}) \\ T_{0} & \text { mean temperature, } T_{0}=\frac{T_{c}+T_{h}}{2}(K) \\ U_{r e f} & \text { reference velocity, } U_{r e f}=\alpha \frac{\sqrt{R a}}{L}\end{array}$

\footnotetext{
${ }^{*}$ Corresponding author, Tel: +335494981 18, Fax: +33549498101

Email addresses: yann.billaud@ensma.fr (Y. Billaud), didier.saury@ensma.fr (D. Saury), denis.lemonnier@ensma.fr (D. Lemonnier)
} 


$\begin{array}{ll}x_{H_{2} O} & \text { molar fraction of water vapor } \\ \text { Dimensionless numbers } \\ N u^{c} & \text { convective Nusselt number, } N u^{c}=\frac{L}{\Delta T}\left(\frac{\partial T}{\partial x}\right)_{x^{*}=0,1} \\ N u^{r} & \text { radiative Nusselt number, } N u^{r}=\frac{L q_{r}^{2 n c}}{\lambda \Delta T} \\ P r & \text { Prandtl number, } P r=\frac{\mu C p}{\lambda} \\ R a & \text { Rayleigh number, } R a=\frac{g \beta \Delta T L_{z}}{\nu a} \\ S & \text { dimensionless stratification parameter, } S=\frac{L}{\Delta T L_{y}} \int_{y^{*}=0}^{1}\left(\frac{\partial T}{\partial z}\right)_{x^{*}=y^{*}=0.5} d y^{*} \\ T^{*} & \text { dimensionless temperature, } T^{*}=\frac{T-T_{0}}{T_{h}-T_{c}} \\ u^{*}, v^{*}, w^{*} & \text { dimensionless velocity components along x, y and z axis, e.g. } u^{*}=\frac{u}{U_{r e f}} \\ x^{*}, y^{*}, z^{*} & \text { dimensionless coordinate, e.g. } x^{*}=\frac{x}{L} \\ \text { Greek symbols } & \text { thermal diffusivity }\left(m^{2} / s\right) \\ \alpha & \text { thermal expansion coefficient, } \beta=\frac{-1}{\rho_{0}} \frac{\partial \rho}{\partial T}(1 / K) \\ \beta & \text { emissivity } \\ \varepsilon & \text { direction cosines } \\ \mu, \eta, \xi & \text { absorption coefficient associated with the } j^{t h} \text { fictitious gray gas }(1 / m) \\ \kappa, & \text { wavelength }(\mu m) \\ \lambda & \text { fluid density }\left(k g / m^{3}\right) \\ \rho & \text { StefanBoltzmann constant, } \sigma=5.670367 \times 10^{-8}\left(W / m^{2} . K^{-4}\right) \\ \sigma & \text { propagation direction, } \Omega \in[0,4 \pi] \\ \Omega & \text { black body } \\ \text { Subscript } & \text { number of direction cosine under consideration, } m \in[1, M] \\ b & \text { number of gray gas under consideration, } j \in\left[0, N_{g}\right] \\ m & \text { wall }\end{array}$

\section{Introduction}

Natural convection in differentially heated cavities has been extensively investigated in the past decades because of its academic nature, well adapted to a wide range of engineering applications, and for understanding the transition phenomena leading to unsteady flows. Benchmark solutions for square cavities were first obtained at low Rayleigh numbers $\left[10^{3}-10^{6}\right]$ by De Vahl Davis and Jones [1] and, later, at higher values $\left[10^{7}-10^{8}\right]$ by Le Quéré [2]. The effects of wall radiation on the flow structure, temperature field and heat transfer were first investigated in two dimensions by Larson and Viskanta [3], and more recently by Nouanegue et al. [4], under the assumption of a fully transparent fluid. A strong influence was found, especially near the top and bottom adiabatic walls (considered as black surfaces) resulting in a decrease of the thermal stratification in the core of the cavity. The same conclusion was drawn in three-dimensionnal configurations [5]. Another specific case of coupling occurs when the fluid absorbs and emits infrared radiation, such as gas mixtures involving water vapour or carbon dioxide, for instance. Significant effects on flow field and heat transfer were observed, since, in this configurartion, radiative transfer directly affects the local energy balance. The first evidences of this phenomenon were found in bidimensionnal simulations and under the assumption of a gray medium by Lauriat [6] then by Yucel et al. [7]. They show that the temperature distribution is strongly modified, which, in turn, enhances the global flow motion. Later, and still using a gray gas model, the effect of optical thickness was studied by Lari et al. [8] and extensions to the case of three-dimensional cavities were conducted by Colomer et al. 
[9], Borjini et al. [10] and Kumar and Eswaran [11]. Although some general trends could safely be derived regarding the role of volume radiation, such as a thickening of boundary layers and an increase of maximum velocities, the gray gas assumption fails to predict the actual behavior of real gases. This issue was first stated by Borget et al. [12], who considered molecular gas radiation to predict the onset of instability and transition to turbulence in a differentially heated cavity. The most recent studies devoted to the interaction between gas radiation and natural convection, whether in square $[13,14]$ or cubic cavities $[9,15,16]$, resort to global models to precisely account for the spectral properties of molecular gases.

A detailed review of the literature shows a lack of knowledge of the effect of the enclosure size on flow structure and heat transfer when gas radiation occurs. The point is that, without gas radiation, natural convection flows in air is fully characterized by the Rayleigh number, providing that the unknown quantitites are set in a correct non-dimensional form (velocities, temperature, heat fluxes). With gas radiation, and in the limit of the gray fluid assumption, additional parameters are needed, such as (i) the ratio of the temperature difference between walls to the average fluid temperature, (ii) a conduction-to-radiation parameter (often termed as the Stark number) and (iii) the overall cavity opacity (the product of the cavity size by the absorption coefficient). This means that the Rayleigh number - which includes size effects - is no longer sufficient to characterize the flow and heat transfer fields, but the actual length of the enclosure has to be specified in addition. This remains true if real (non-gray) gas radiation is considered, knowing that in that case it is impossible to characterize the configuration by a finite set of non-dimensionnal parameters. The reason is that the aborption spectrum itself involves about one million lines, whose variation cannot be described in a general shape with a limited number of parameters only, even for compact models. Therefore, when working with humid air, temperature, pressure and concentration have to be fixed, which in turn prescribes the values of all the transport properties, and physical similarity non longer exists. Nevertheless, the Rayleigh number remains a pertinent parameter, especially for comparison with solutions where gas radiation is neglected. This is probably why some recent studies in humid air, including gas radiation effects, have drawn conclusions (transition to unsteadyness and turbulence, for instance) in terms of $R a$ values only. The purpose of this study is therefore to demonstrate that, even at a given $R a$, the flow may display different characteristics if the size of the cavity is varied.

We consider a 3D adiabatic differentially heated cubic cavity filled with an air $/ \mathrm{H}_{2} \mathrm{O}$ mixture, representative of building applications. The numerical simulations are carried out considering either wall, gas, or both wall and gas radiation, and are compared to solutions obtained without any radiative coupling. When the gas is assumed to be a participating medium, a global model is used for treating the infrared radiation of water vapour. In the present steady-state laminar flow study, we will focus on a unique value of Rayleigh number, far below the transition to unsteadiness in an adiabatic cubic cavity without radiation $R a_{c}=3.2 \times 10^{7}[17]$ in order to emphasize radiation effects. The paper is organized as follows. In a first section, after an overview of the physical problem under consideration, the governing equations of the coupled problem are reminded, as well as the global model we used for the gas radiative properties. Then, we present in detail the methods implemented for numerical simulations, followed by validation of the coupling methodology by comparison with benchmark solutions. Results of coupled simulations are then analyzed for $R a=10^{6}$ at different heigths of the cavity $(L=1,2,3 \mathrm{~m})$. We especially focus on this size effect on the structure of the thermal and dynamic fields, on heat transfer, and on 3D patterns for four different configurations. 


\section{Mathematical model}

\subsection{Problem statement}

A differentially heated cubic cavity of variable height is filled with a mixture of dry air and water vapour $\left(x_{\mathrm{H}_{2} \mathrm{O}} \approx 10^{-2}\right)$. This globally corresponds to ambient conditions at $50 \%$ of humidity. The two facing vertical walls are considered as black isothermal surfaces, having different temperatures, $T(x=0)=T_{h}$ and $T(x=L)=T_{c}$. The other four walls are adiabatic (Fig. 1) and - depending on the test case - either black or diffuse and purely reflecting. In order to separate the effects of gas and wall radiation, and further study their combined effects, four distinct configurations are investigated, as summarized in Table 1.

In case $\mathrm{A}$, the adiabatic walls are purely reflecting and gas radiation is disregarded: in this situation, radiative transfer and convection are uncoupled phenomena. In case B, gas radiation is considered alone: it induces an additional source term in the energy budget of the fluid, due to the local balance between volume absorption and emission. In case $\mathrm{C}$, surface radiation from the adiabatic walls is considered, without any radiative participation of the gas (transparent): the zero-flux condition along passive walls prescribes that radiation gained by the solid surface must be compensated by an equivalent convective loss. This creates a strong coupling with the thermal field through the boundary conditions along these walls. Finally, in case D, both gas and wall radiation effects are combined.

In this work, the buoyancy-driven flow is generated by imposing a temperature difference between the two isothermal walls. This difference is adapted to the cavity size so that the Rayleigh number is kept at a same value, $R a=10^{6}$, whatever the size $L$. The relatively low temperature difference encountered in the present work makes the Boussinesq approximation entirely legitimate. Calculations are performed in transient regime but only steady-state solutions are presented in this work.

\subsection{Conservation of mass, momentum and energy}

The governing equations, namely the conservation of mass, momentum and energy, may be expressed, under the Boussinesq approximation, as

$$
\begin{aligned}
\frac{\partial u}{\partial x}+\frac{\partial v}{\partial y}+\frac{\partial w}{\partial z} & =0 \\
\frac{\partial u}{\partial t}+u \frac{\partial u}{\partial x}+v \frac{\partial u}{\partial y}+w \frac{\partial u}{\partial z} & =-\frac{1}{\rho} \frac{\partial p_{m}}{\partial x}+\nu\left(\frac{\partial^{2} u}{\partial x^{2}}+\frac{\partial^{2} u}{\partial y^{2}}+\frac{\partial^{2} u}{\partial z^{2}}\right) \\
\frac{\partial v}{\partial t}+u \frac{\partial v}{\partial x}+v \frac{\partial v}{\partial y}+w \frac{\partial v}{\partial z} & =-\frac{1}{\rho} \frac{\partial p_{m}}{\partial y}+\nu\left(\frac{\partial^{2} v}{\partial x^{2}}+\frac{\partial^{2} v}{\partial y^{2}}+\frac{\partial^{2} v}{\partial z^{2}}\right) \\
\frac{\partial w}{\partial t}+u \frac{\partial w}{\partial x}+v \frac{\partial w}{\partial y}+w \frac{\partial w}{\partial z} & =-\frac{1}{\rho} \frac{\partial p_{m}}{\partial z}+\nu\left(\frac{\partial^{2} w}{\partial x^{2}}+\frac{\partial^{2} w}{\partial y^{2}}+\frac{\partial^{2} w}{\partial z^{2}}\right)+g \beta\left(T-T_{0}\right) \\
\frac{\partial T}{\partial t}+u \frac{\partial T}{\partial x}+v \frac{\partial T}{\partial y}+w \frac{\partial T}{\partial z} & =\alpha\left(\frac{\partial^{2} T}{\partial x^{2}}+\frac{\partial^{2} T}{\partial y^{2}}+\frac{\partial^{2} T}{\partial z^{2}}\right)-\frac{1}{\rho c_{p}} \nabla \cdot \mathbf{q}_{\mathbf{r}}
\end{aligned}
$$

The thermophysical properties of air, considered to be unaffected by the presence of a (very small) amount of water vapour, are evaluated at the reference temperature $T_{0}=\frac{1}{2}\left(T_{c}+T_{f}\right)$ from Ref. [18]. The radiative source term $-\boldsymbol{\nabla} \cdot \mathbf{q}_{\mathbf{r}}$ in equation 5 is calculated by

$$
-\nabla \cdot \mathbf{q}_{\mathbf{r}}=\int_{0}^{\infty} \kappa_{\lambda}\left[G_{\lambda}-4 \pi I_{b, \lambda}\right] d \lambda
$$


where $\kappa_{\lambda}$ is the spectral absorption coefficient of the gas, $I_{b, \lambda}$ the spectral blackbody intensity and $G_{\lambda}$ the spectral incident radiation. This last quantity is evaluated by the integral $G_{\lambda}=\int_{4 \pi} I_{\lambda} d \Omega$, once the intensity fields are known - as solutions of the radiative transfer equation (RTE) (see equation 7 below) - in all directions and over the whole spectrum.

\subsection{Radiative Transfer Equation}

The RTE describes the propagation of spectral radiation across the gaseous domain in one given direction s. Its expression, for a non-scattering medium (no solid particles within the gas), is

$$
\mu \frac{\partial I_{\lambda}}{\partial x}+\eta \frac{\partial I_{\lambda}}{\partial y}+\xi \frac{\partial I_{\lambda}}{\partial z}=-\kappa_{\lambda} I_{\lambda}+\kappa_{\lambda} I_{b, \lambda}
$$

In this equation, $I_{\lambda}$ is the local value of the spectral intensity in direction $\mathbf{s},(\mu, \eta, \xi)$ are the direction cosines of $\mathbf{s}$, and $\kappa_{\lambda}$ is the local spectral absorption coefficient. In our configuration, the total pressure and the molar fraction of $\mathrm{H}_{2} \mathrm{O}$ are uniform over the domain. Therefore, the values of $\kappa_{\lambda}$ varies with temperature only. To determine the total (spectrally integrated) intensity field, the RTE has to be solved, in theory, for every possible wavelength $\lambda$. The most accurate method at our disposal is the line-by-line (LBL) calculation: it, however, necessitates up to $10^{5}-10^{6}$ discrete value of $\lambda$ to correctly represent the strong variations of $\kappa_{\lambda}$ over the spectrum, and as many solutions of the RTE (for each values of $\lambda$ ). This requires excessive computer resources for practical applications and, especially, for coupled problems. On the other hand, compact methods have been developed in the past 20 years (and continuously improved since then), such as the SLW model by Denison and Webb [19]. This model is based on some statistical reordering of the absorption spectrum, and only about 10 discrete values of the spectral variable (no longer exactly a wavelength) are needed. For homogeneous gases (isothermal, uniform composition), the SLW model displays almost the same level of accuracy as LBL, but errors may be larger in presence of strong temperature gradients, like in combustion problems, for instance. However, since our configuration involves only weak temperature variations, SLW has been chosen to model the spectral behaviour of the air- $\mathrm{H}_{2} \mathrm{O}$ mixture in the cavity.

\subsection{Spectral Model}

In the SLW model (Spectral Line Weighted sum of gray gases), developed by Denison and Webb $[20,19,21]$, the non-gray gas mixture is replaced by a set of $N_{g}$ fictitious gray gases, plus one clear (transparent) gas. The characteristics of these gases are obtained by evenly dividing on a logarithmic scale the range of absorption cross-section values $\left[\widetilde{C}_{a b s, \min } ; \widetilde{C}_{a b s, \text { max }}\right]$ for the radiating species under consideration $\left(\mathrm{H}_{2} \mathrm{O}\right)$. Then, the $j^{\text {th }}$ gray gas is given a $C_{a b s}$ - value, which is the geometric mean of two adjacent discrete values : $C_{a b s, j}=\sqrt{\widetilde{C}_{a b s, j-1} \widetilde{C}_{a b s, j}}$. The relevant absorption coefficient is further obtained by multiplying the cross-section by the gas molar density. For water vapor, considered as a perfect gas, this amount correspond to $\kappa_{j}=C_{a b s, j} \times x_{H 2 O} P / R T$, where $P$ is the total pressure and $R$ the universal gas constant $\left(R=8.314510 \mathrm{~J} \mathrm{~mol}^{-1} \mathrm{~K}^{-1}\right)$. Each gray gas is also attributed the weighting factor

$$
a_{j}=F\left(\widetilde{C}_{a b s, j}\right)-F\left(\widetilde{C}_{a b s, j-1}\right)
$$

where $F$ is defined as the fraction of the blackbody emission - at local temperature $T$ - over wavelengths where the absorption cross-section - at the reference state $T_{0}, P, x_{\mathrm{H} 2 \mathrm{O}}$ - is less than a certain value $C_{a b s}$

$$
F\left(C_{a b s}\right)=\frac{1}{\sigma T^{4}} \int_{C_{a b s, \lambda}<C_{a b s}} I_{b, \lambda}(T) d \lambda
$$


In the present work, the correlation proposed by Denison and Webb [19] was used to evaluate this $F$-function for water vapour and, in turn, calculate the weights $a_{j}$ for all but the transparent component $(j=0)$. For the latter, we simply set $\kappa_{0}=0$ and, due to the natural normalisation constraint, $a_{0}=1-\sum_{j=1}^{N_{g}} a_{j}$.

Finally, the RTE to be solved for each gray gas takes the form

$$
\mu \frac{\partial I_{j}}{\partial x}+\eta \frac{\partial I_{j}}{\partial y}+\xi \frac{\partial I_{j}}{\partial z}=-\kappa_{j} I_{j}+a_{j} \kappa_{j} \frac{\sigma T^{4}}{\pi}, \quad j=0, \cdots, N_{g}
$$

and the total intensity can further be found by adding the contribution of each solution $I_{j}$ over all gray gases: $I=\sum_{j=0}^{N_{g}} I_{j}$.

\section{Numerical method}

In this section, the solution method for the radiation problem, as well as its coupling with the general purpose CFD software Code_Saturne [22] computing the flow motion and the temperature field (Eqs. 1-5) are described.

\subsection{RTE Solution}

In the present work, the Discrete Ordinate Method (DOM) [23] have been used to solve the RTE for each gray gas of the SLW model. This method consists in solving the transport equation over a discrete set of directions $\left(\mu_{m}, \eta_{m}, \xi_{m}\right)$ and then calculate by quadrature formulae the integrals over directions involved in the definition of the radiative flux and source terms. This method, coupled with the SLW approach, yields the following expression of the radiative source

$$
\boldsymbol{- \nabla} \cdot \mathbf{q}_{\mathbf{r}}=\sum_{j=0}^{N_{g}}\left[\kappa_{j} \sum_{m=1}^{M} w_{m} I_{j, m}-4 a_{j} \sigma T^{4}\right]
$$

where $w_{m}$ is the weight attributed to the $m$-th direction and $M$ the total number of discrete directions.

Similarly, the radiative incident flux at the wall can be expressed as

$$
\begin{aligned}
q_{r, j}^{i n c} & =\left[\kappa_{j} \sum_{m=1 \& \mathbf{n} \cdot \mathbf{s}_{\mathbf{m}}<0}^{M} w_{m} s_{m} I_{j, m}\right], \quad \text { contribution of the } j^{t h} \text { gray gas, } \\
q_{r}^{i n c} & =\sum_{j=0}^{N_{g}} q_{r, j}^{i n c}, \quad \text { total (spectrally integrated) value. }
\end{aligned}
$$

\subsection{Boundary conditions}

A no-slip condition is imposed on each wall (Fig. 1). Both active walls are assumed to be black and isothermal surfaces, whose temperature is prescribed at $T_{h}(x=0)$ and $T_{c}<T_{h}(x=L)$. The remaining (passive) four walls are adiabatic and considered either as blackbodies $\left(\varepsilon_{w}=1\right)$ or perfectly diffuse reflecting $\left(\varepsilon_{w}=0\right)$. Boundary conditions for temperature on passive wall may be globally expressed as

$$
\varepsilon_{w}\left(\sigma T_{w}^{4}-q_{r}^{i n c}\right) \pm \lambda\left(\frac{\partial T}{\partial n}\right)_{w}=0
$$


where $n$ denotes the unit vector normal to the surface and pointing inward the medium. When $\varepsilon_{w}=0$, this condition simply reduces to $\frac{\partial T}{\partial n}=0$. Otherwise, it expresses a transfer of energy between convection and radiation at the wall, so that to preserve the zero net flux condition.

Whatever the wall (active or passive), the boundary condition for intensity related to the $j^{\text {th }}$ gray gas of the SLW model is

$$
I_{j}^{w}=\varepsilon_{w} a_{j} \frac{\sigma T_{w}^{4}}{\pi}+\frac{1-\varepsilon_{w}}{\pi} q_{r j}^{i n c}
$$

\subsection{Coupling and numerical solution procedure}

All the CFD computations have been carried out using the Code_Saturne software [22], in which are implemented our own radiation modules (discrete ordinate method and SLW model). A Second Order Linear Upwind Scheme (namely SOLU) was used for the momentum equations and a centred and conservative second order scheme in space was applied to the energy equation. Finally, the temporal discretisation was based on an implicit first-order Euler method. The pressurevelocity coupling was treated by the SIMPLEC algorithm and a gradient reconstruction method was introduced to properly predict the heat transfer between the walls and the fluid. The intensity field was calculated by solving the radiative transfer equation (10), for each gray gas, with temperature values taken at the previous time step. An inner iteration loop is used to meet the boundary condition on intensity (15) when walls are reflecting.

The overall procedure may be summarized as follow:

1. Initialization of the velocity, temperature and radiation intensity fields,

2. Solution of the RTE for a given temperature field, for each gray-gas coming from the SLW model,

3. Evaluation of the radiative volumetric source $\left(-\boldsymbol{\nabla} \cdot \mathbf{q}_{\mathbf{r}}\right)$, further introduced as a source term in the energy equation (5),

4. Calculation of the velocity fields by solving the coupled set of equations (1-4), with temperature values coming form the previous time step.

5. Update of the temperature field, by solving the energy equation (5)

6. Repeat steps 2 to ?? until steady-state is reached, according to the criterion $\max \left|\left(\Phi_{P}^{n}-\Phi_{P}^{n-1}\right) /\left(\Phi_{\text {ref }}\right)\right| \leq 10^{-4}$, where $\Phi$ stands for $u, v, w$ or $T$ and $\Phi_{\text {ref }}$ is either $\alpha \sqrt{R a} / L_{z}$ for velocity components or $\left(T_{h}+T_{c}\right) / 2$ for temperature.

\section{Code validation}

The accuracy of the whole simulation code was checked with respect to:

1. the direction quadrature used with the DOM to solve the radiative transfer equation;

2. the number of fictitious gray gases involved in the SLW model;

3. the spatial grid size and distribution.

\subsection{DOM validation and convergence with respect to the quadrature set}

Validation of the radiative part of the code is carried out against data provided by Soucasse et al. [24]. The 3D test case consists of a cubic cavity of length $L=1 \mathrm{~m}$ containing a gray medium, whose absorption coefficient is set to $\kappa=1 \mathrm{~m}^{-1}$. All the walls are isothermal $\left(T_{0}=300 \mathrm{~K}\right)$, gray and diffuse with an emissivity of $\varepsilon_{w}=0.5$. The temperature distribution within the medium is prescribed as $T(x, y, z)=\exp \left[-\left(x-x_{0}\right)^{2}-\left(y-y_{0}\right)^{2}-\left(z-z_{0}\right)^{2}\right] \Delta T+T_{0}$, with $x_{0}=y_{0}=z_{0}=0.25$ 
and $\Delta T=10 \mathrm{~K}$. The radiative equilibrium in this configuration was calculated by Soucasse et al. [25] by using a Monte Carlo Method on a uniform grid of $42^{3}$ cells. Here, the DOM is used with the $S_{6}, S_{8}$ and $S_{10}$ quadratures, involving 48, 80 and 120 discrete directions, respectively. The comparison is made in terms of radiative flux $q_{w}$ and radiative volumetric power $\boldsymbol{\nabla} . \mathbf{q}_{\mathbf{r}}$ along different $x$-lines inside the cavity. As illustrated in Fig. 2, a very good agreement is obtained (Fig. $2 \mathrm{a}$ and Fig. $2 \mathrm{~b}$ ) and the $S_{8}$ quadrature was found sufficient to keep relative errors below $1 \%$.

\subsection{Validation of the SLW approach and convergence with respect to the number of gray gases}

Only few benchmarks exist for the validation of $3 \mathrm{D}$ radiative transfer codes that take into account the spectral properties of the medium. We have considered the configuration proposed as reference by Liu [26], which consists of a rectangular cavity of $2 m \times 2 m \times 4 m$ containing pure water vapor at uniform temperature of $1000 \mathrm{~K}$ surrounded by black walls at $300 \mathrm{~K}$.

The solution was obtained using a ray tracing method and a statistical narrowband model (SNB). Our calculation were performed using the DOM with a $S_{8}$ quadrature and using the SLW model with a varying number of gray gases: $N_{g}=8$ and 20 . The gas absorption cross-sections were taken in the range $\left[3 \times 10^{-5} ; 120\right] \mathrm{m}^{2} . \mathrm{mol}^{-1}$, which is further divided into $N_{g}+1$ discrete values, $\widetilde{C}_{a b s, j}$, evenly spaced over a logarithmic scale. Then, the cross-section attributed to the $j^{\text {th }}$ gray gas is evaluated as $C_{a b s, j}=\sqrt{\widetilde{C}_{a b s, j-1} \widetilde{C}_{a b s, j}}$. Calculations were performed using the same uniform grid as in Ref. Liu [26], i.e. $11 \times 11 \times 16$. Comparisons are presented in Fig. 3(a-d) in terms of radiative heat source and incident fluxes.

With $N_{g}=8$ gray gases, radiative sources $\left(-\nabla \cdot \mathbf{q}_{\mathbf{r}}\right)$ are slightly overpredicted $(3 \%)$ in most of the domain (Fig. 3a and 3b). The largest differences $(6 \%)$ are observed close to the side walls ; they are mainly induced by the temperature drop prescribed between the medium (1000 K) and the bounding surfaces $(500 \mathrm{~K})$. Such discontinuities do not occur in more realistic situations, especially when coupled heat transfer prevails. On the other hand, incident heat fluxes $\left(q_{w}\right)$ are well predicted as the relative error does not exceed $2 \%$, whatever the position (Fig. 3b and 3d). Moving to $N_{g}=20$ does not significantly improve the computational accuracy.

Finally, we have used in this study the discrete ordinates method with the $S_{8}$ quadrature and radiative properties of humid air are taken into account by the SLW model considering 8 fictitious gray gases (+1 clear).

In addition, it is worth pointing out that we use the SLW data taken from Ref. [20], even for applications at temperature below the range where the correlations for function $F$ were derived $[400-2500 K]$. However, we ensured these correlations still return accurate result at $T=300 K$, which is not the case with new correlations proposed by Pearson et al. [27], the latter being on the other hand more precise at high temperature.

\subsection{Final code validation}

In this last validation test, a $1 \mathrm{~m}^{3}$ cubic cavity filled with non gray air- $\mathrm{H}_{2} \mathrm{O}$ homogeneous mixture is investigated under atmospheric pressure and at an initial mean temperature $T_{0}=300 \mathrm{~K}$. The molar fraction of water vapor is $x_{\mathrm{H}_{2} \mathrm{O}}=0.115$ and we consider that such a small amount does not affect the thermal properties of air : $\lambda=0.0263 W \cdot m^{-1} \cdot K^{-1}, \alpha=2.25 \times 10 \mathrm{~m}^{2} \cdot \mathrm{s}^{-1}$ and $\operatorname{Pr}=0.707$. The temperatures of the two active walls are set to $T_{h}=T_{0}+\Delta T / 2$ and $T_{c}=T_{0}-\Delta T / 2$, respectively, and the value of $\Delta T$ is selected as $\Delta T=0.011 \mathrm{~K}$ in order to prescribe, according the cavity size, the Rayleigh number at $R a=10^{6}$. Four different configurations are investigated, whose characteristics are summarized in Table 1.

In case A and case C, a fictitious fluid is considered, having the same properties as the air- $\mathrm{H}_{2} \mathrm{O}$ mixture, but remaining totally transparent to infrared radiation. Gas radiation is cancelled out 
and the radiative source term in the energy equation disappears. Moreover, in case A, the passive walls are purely reflecting $\left(\varepsilon_{w}=0\right)$, meaning that there is no coupling with radiation through the adiabatic conditions (15). Therefore, in case $\mathrm{A}$, the radiative transfer exists - between the two active walls - but is totally uncoupled from the flow motion and convective heat transfer in the cavity. In case $\mathrm{C}$ the radiative coupling exists, but only with surface radiation, through the boundary conditions. In case $\mathrm{B}$ and $\mathrm{D}$, the gas radiation is considered. The source term $\mathbf{- \nabla} \cdot \mathbf{q}_{\mathbf{r}}$ in the energy equation is calculated as a balance between the incoming (molecular absorption) and outgoing (molecular emission) radiant energies. In case B, only the gas radiation is considered (no surface radiation effects). In case D, both gas and wall radiation couplings are considered.

In order to validate our code, the results from the four test cases were compared against data taken from Ref. [24]. In this reference, a ray tracing method was used to solve the RTE, along with the ADF model for gas radiation (quite similar to SLW) and a spectral Chebyshev collocation method [28] for the resolution of the flow equations; calculations were performed over a $81^{3}$ point mesh within the flow and $40^{3}$ points for the radiation field. In our simulations, the mesh is the same for both the flow and radiative transfer, namely $91^{3}$ points with a non uniform distribution along the three axes whose density is given by $d=\cosh (6 t-3)$ with $t \in[0 ; 1]$. In this validation case study, the cell size ranges between $1.85 \mathrm{~mm}$, close to the walls, and $36.20 \mathrm{~mm}$, in the middle of the cavity. Comparison between our calculations and the reference is made in terms of temperature profiles along the x-axis on the top wall (Fig. 4a) and along the z-axis in the middle of the cavity (Fig. 4b). As in Ref. [24], the profiles are averaged over $y^{*}$.

The agreement between the reference and our model predictions, both for temperature profile along the center line or at the top wall, is excellent. The validation is completed by a comparison in terms of velocity profiles along $\mathrm{x}$ at three different heights (Fig. 5), $z^{*}=[0.25 ; 0.5 ; 0.75]$.

The plots on Fig. 5 show that the vertical velocities are nearly the same for both calculations, even if the solution is obtained about 10 times faster in the present work.

Although not presented here, a sensitivity study was performed to analyse the influence of the mesh spacing on the convective and radiative Nusselts and on the stratification parameter. It was observed that increasing the size of the mesh (i.e. $111^{3}$ ) does not affect significantly the results $(<1 \%)$, whatever the configuration. These results motivate the selection of the $91^{3}$ mesh as fine enough to perform the following numerical studies.

The solutions obtained with this mesh are then compared with the reference solutions [24] in Table 2 in terms of average convective and radiative Nusselt numbers on the hot wall, stratification parameter $S$ (vertical temperature gradient at $x^{*}=z^{*}=0.5$, averaged over y) and average temperature at the top wall $\theta_{\text {top }}$ for $R a=10^{6}$, for the four cases under investigation.

In case $\mathrm{A}$, the flow and the convective heat transfer are well predicted: the relative error is less than $1 \%$ for each parameters, except the radiative Nusselt number (10\%). A Monte Carlo method is then used to separately evaluate the radiant exchanges between the isothermal vertical walls. The radiative Nusselt number was found to converge to 125.4, a value close to 125.6 as found by the present model. Note that the value provided by Soucasse et al. [24] (139.7) is based on a global radiosity calculation (one single node per wall), which is not accurate enough in this configuration.

In case B, all quantities compare well with Ref. [24] and the relative error does not exceed $2 \%$. The small discrepancies may result from the fact that $\mathrm{CO}_{2}$ is not taken into account in the present study.

In case $\mathrm{C}$, the adiabatic walls absorb the incident radiation, whose magnitude is stronger close to the hot surface. A refined mesh in this zone allows a more accurate prediction of the wall temperature, which has a predominant effect on the flow structure. The same observation holds near the cold wall. The use of a refined radiative mesh may explain the discrepancy of about $4 \%$ with Ref. [24] on the convective Nusselt number. The discrepancy concerning the stratification 
parameter (4\%) is due to the relatively coarse mesh in the cavity core (Fig.1).

In case $\mathrm{D}$, where the effects described for cases $\mathrm{B}$ and $\mathrm{C}$ are cumulated, the maximum difference with respect to reference data is $5 \%$ for the convective Nusselt number and $8 \%$ for the stratification parameter (with still the central mesh in question). On the other hand, the average top wall temperature $\theta_{\text {top }}$ is always well predicted as the relative difference is lower than $1 \%$ whatever the case.

\section{Results and discussion}

In this study, the four specific cases presented previously (Table 1) are considered. The effect of the cavity size is investigated while keeping the Rayleigh number at a same value, $R a=10^{6}$, and with data representative of building applications. The mean temperature is chosen as $T_{0}=$ $293.15 K$. The density, viscosity, heat capacity and thermal conductivity are set, respectively, to $1.205 \mathrm{~kg} \cdot \mathrm{m}^{-3}, 1.82329 \times 10^{-5}$ Pa.s, $1005 \mathrm{~J} . \mathrm{kg}^{-1} \cdot \mathrm{K}^{-1}$ and $0.0257 \mathrm{~W} \cdot \mathrm{m}^{-1} \cdot \mathrm{K}^{-1}$. The relative humidity is $50 \%$, which, under the prescribed conditions and at atmospheric pressure, yields a water vapor molar fraction of $x_{H_{2} O}=0.0115$. The domain is meshed using $91^{3}$ points, both for the flow and radiation fields. This mesh, as well as the DOM quadrature set $\left(\mathrm{S}_{8}\right)$ and the number of gray gases in the SLW model $\left(N_{g}=8\right)$, were previously shown to be sufficient for predicting the global flow features and heat transfer results in this configuration. The time step has to be set in order to take into account all the time scales associated with conduction $\left(\tau_{c}=\rho c_{p} L^{2} / \lambda\right)$, buoyancy $\left(\tau_{b}=\sqrt{L / g \beta \Delta T}\right)$ and viscosity momentum transfer $\left(\tau_{v}=L^{2} / \nu\right)$. All of them depend on the size of the domain $L$. However, whatever the length used in this study, the shortest time scale remains the convective (buoyancy) one. Therefore, our computational time step was set to $\Delta t=1 \mathrm{~s}$, which is two order of magnitude smaller than the minimum convective time scale encountered (for $L=1 \mathrm{~m}$ ). Moreover, since the time scale associated to radiation equilibrium $\left(\tau_{r}=\rho c_{p} L \Delta T / \sigma T_{0}^{4}\right)$ is extremely short compared to the convective one, it was found sufficient to update the radiative source terms every 10 time steps only.

As a first step, the flow structure and thermal field in the mid-plane $z=L / 2$ are observed. For comparison purpose, the dimensionless coordinates $x^{*}, y^{*}, z^{*}$ related to the cavity length $L$ are introduced, as well as dimensionless velocities $\left(u^{*}, v^{*}, w^{*}\right.$ related to $\left.U_{\text {ref }}=\alpha \sqrt{R a} / L\right)$ and temperature $\left(T^{*}=\left(T-T_{0}\right) / \Delta T\right)$. In addition, the global heat transfer (convective and radiative), as well as the stratification parameter or maximum velocities, are examined and the $3 \mathrm{D}$ effects are discussed. In this study, the cavity height ranges from $1 \mathrm{~m}$ to $3 \mathrm{~m}$.

\subsection{Airflow structure and velocity profiles}

As a first approach, we focus on the mid-depth $\left(y^{*}=0.5\right)$ plane solutions, where velocity vectors are plotted for each case under consideration (see Fig. 6).

In case A (no radiative coupling), the velocity field displays typical patterns, i.e. a centrosymmetric flow with separate boundary layers and relatively low velocities, especially in the upper hot lower cold side corners of the cavity. A hydraulic jump is observed relatively close to the adiabatic walls. Along the cavity roof, the separation point, located at $x^{*} \approx 1 / 3$, is mainly due to the impact of the thin ascending boundary layer on the top wall combined with a strong vertical thermal stratification. As expected, when radiation is disregarded, the change in cavity size does not affect the flow topology. But when gas radiation is accounted for (case B), the viscous boundary layers get thicker, mainly in the upper half of the hot wall (and in the lower half of the cold wall) and the fluid is accelerated in the whole cavity (Table 3). Then, increasing the cavity size mitigates the hydraulic jump, moves it toward the opposite vertical wall and enhances the horizontal wall jets. 
Note that the flow topology also changes from a separate boundary-layer structure to a rotating core flow when $L$ is increased.

When the fluid is considered as transparent and all the walls behave as black radiating surfaces (case $\mathrm{C}$ ), the flow structure is mainly affected along the top and bottom boundaries. As observed in Fig. 4, the upper wall temperature is decreased, which in turn lowers the near-wall fluid temperature and limits the appearance of a hydraulic jump. Unlike case B, increasing the cavity length does not affect the flow since radiation view factors between all walls remain the same (the aspect ratio is preserved), and therefore the temperature distribution along the adiabatic wall temperature does not change.

The combination of wall and gas radiation (case D) cumulates the effects previously described in case B and C. As already observed [24], wall radiation magnifies the effect of gas radiation on the flow structure. These mechanisms result in the formation of a large cell driving the fluid in the core of the cavity. Case D also leads to the higher velocities (Table 3), and, for the largest cavity under consideration, $L=3 \mathrm{~m}$, to a rotating flow.

For cases $\mathrm{B}$ and $\mathrm{D}$, the larger the cavity, the more intense the flow is. This observation proves that a criterium only based on the Rayleigh number may not be sufficient to predict the transition between laminar, transitional and turbulent flows.

Fig. 7 shows the profiles of vertical and horizontal velocity, both averaged over $y^{*}$, at $z^{*}=0.5$ and $x^{*}=0.5$. Once again, the effects of both gas (case $\mathrm{B}$ ) and wall radiation (case $\mathrm{C}$ ) on the global flow are noticeable, yielding an overshoot of the vertical and, to a greater extent, of the horizontal components. When gas radiation is considered alone (case B), increasing the cavity size produces an increase of the maximum velocity, a thickening of the boundary layers and an acceleration of the flow in the core of the cavity. These phenomena are all the more important when gas radiation and wall radiation are simultaneously considered (case D). One should notice that, even in the cases with gas radiation, yet known to break symmetries, the centrosymmetry of the thermal and flow fields is essentially preserved, because of the small temperature difference involved in this problem (and radiation effect may somehow be linearized). This observation holds regardless the cavity size, at least within the range of dimension and temperature considered here.

\subsection{Temperature field}

Iso-values of the temperature in the plane $y^{*}=0.5$ (Fig. 8) and temperature profiles, averaged over y at $z^{*}=0.5$ and $x^{*}=0.5$ (Fig. 9), confirms the observations previously drawn regarding cavity size dependency, whatever the case.

When the gas is transparent (case A), the flow structure previously encountered (large single cell) can again be well identified (Fig. 8). The thermal boundary layers (defined as the distance to the wall where the temperature reaches almost the core temperature) are about 0.2 thick at mid-height of the isothermal walls (Fig. 9a). A slight cooling is observed at $x^{*}=0.1$ due to the transport of cold fluid by the external part of the boundary layer. For symmetrical reasons, a slight heating up is observed at $x^{*}=0.9$. A vertically stratified distribution of temperatures is established in the core, which explains the presence of motionless fluid in the cavity core.

In case $\mathrm{B}$, the temperature field is affected by radiation because the optical thickness changes. In Fig. 8, isotherms 0.2 and 0.4 are mingled whatever the value of $L$, but the slight cooling described previously in case A disappears and the thermal boundary layer thickens with cavity length (Fig. $9 \mathrm{~b})$. The most significant variations occur near the adiabatic walls, where the fluid is precooled by emission along the top wall and preheated by absorption along the bottom one (see Fig. 13). Therefore, increasing the cavity length enhances the effects of radiation which, in turn, tend to homogenize temperature in the cavity. This results in a decrease of the stratification parameter as 
a function of $L$ (Table 4). This trend is confirmed by observation of the thermal vertical distribution at mid-width (Fig. 9).

As previously mentioned, wall radiation (case C) significantly changes the temperature distribution along the adiabatic walls and, consequently, all the thermal field in the flow (when compared to case A). It more specifically decreases the thermal stratification in the cavity centre (Table 4 and Fig. 9). But, unlike the case of gas radiation, changing the cavity length has no effect on temperature distribution.

The cumulative effects of gas and wall radiation (case D) lead however to fewer modifications of the thermal field when the cavity length is varied. Indeed, the temperature of the upper adiabatic wall temperatures is lower when surface radiation is present (case A and B) but this temperature is hardly affected by the size of the cavity (see $\theta_{\text {top }}$, Table 4 ). For this reason, the upper wall jet is only weakly sensitive to cavity dimension.

\subsection{Heat transfer}

Heat transfer is investigated by using the global convective and radiative Nusselt numbers at the hot wall respectively. These parameters are defined as $N u^{c}=\frac{L}{\Delta T} \int_{0}^{1} \int_{0}^{1}\left|\frac{\partial T}{\partial x}\right|_{x^{*}=0} d y^{*} d z^{*}$ and $N u^{r}=\frac{L}{\lambda \Delta T} \int_{0}^{1} \int_{0}^{1}\left|q_{r}^{n e t}\right|_{x^{*}=0} d y^{*} d z^{*}$, respectively, with $q_{r}^{n e t}=\varepsilon_{w}\left(\sigma T_{w}^{4}-q_{r}^{i n c}\right)$. Their values are listed in Table 5.

Gas radiation (case B) affects the amount of heat transported by convection and leads to a decrease of $N u^{c}$ of about $13 \%$ for $L=1 \mathrm{~m}$. This decrease is due to two distinct effects. First, the homogenization of temperature by radiation in the boundary layers decreases the thermal gradient at the wall and thus the local convective Nusselt number. But the main effect seems to be attributed to the horizontal jets along the upper and lower boundaries that significantly affect the convective impact on vertical walls (case A in Fig. 10 and Fig. 11). Cavity length strongly affects the jet structure and, to a lesser extend, temperature which tends to decrease (Fig. 9). As the cavity length increases, the jet become thicker and its trajectory is moving away from the wall. This explains that the maximum value of convective Nusselt number is moving upward to reach the elevation $z^{*} \approx 0.25$. Radiation also modifies the shape of the jet that splits up from $L \geq 2 \mathrm{~m}$ in case B (Fig. 11). 3D effects are discussed in subsection 5.4.

When the adiabatic walls are black (cases $\mathrm{C}$ and $\mathrm{D}$ ), gas radiation does not modify significantly the heat brought by convection $(<1 \%$ for $L=1 \mathrm{~m})$ since the horizontal circulation is already established. However, regarding the effect of cavity length, the same behavior is observed than in case B and leads to an increase in convective Nusselt number. It should be noticed that in case C, $N u^{c}$ should not be modified with cavity length as the geometrical configuration remains the same. The variation of this parameter $(\approx 1.5 \%$ between $L=1 \mathrm{~m}$ and $L=3 \mathrm{~m})$ is attributed to the difficulties for increasing dimensions with constant number of cell to capture the high temperature gradient in the vicinity of the adiabatic black walls (Fig. 10). This may explain the underestimation of $N u^{c}$ in the validation case (Table 2).

The amount of convected heat is also conditioned by the fluid circulation in the upper part of the hot wall (and in the lower part of the cold wall). Observation of the dimensionless vertical velocities $w^{*}$ in Fig. 11 shows a thickening of the boundary layer with cavity length, which induces a larger horizontal jet when gas radiation is only considered (case B). As a result, the increase in cavity length leads to an earlier change in direction of the boundary-layer flow and therefore to an extension of the low-velocity region close to the corners that explains the relatively low value of $N u^{c}$ in the top (or bottom) part of the hot (or cold) wall. The same observation can be drawn in a participating medium when wall radiation is taken into account (case D, Fig. 12).

The average temperature along the top wall $\theta_{\text {top }}$ is constant with length, except in case B, where it decreases. This is due to the increase in horizontal velocity $u_{\max }^{*}$ and the mitigation of 
radiative exchanges with isothermal walls due to increasing optical thickness. Probably the same effects would be observed in case $\mathrm{D}$, but $\theta_{\text {top }}$ is already close to 0 due to wall radiation.

The surface-averaged radiative Nusselt number $N u^{r}$ is used for comparing radiation heat exchange to the reference heat conduction in the cavity but is not appropriate to study the effect of cavity length. The reason is that, the net radiative flux transfered from the hot wall (1) to the cold wall (2) may be expressed as $\left(q_{r}^{\prime \prime}{ }^{\prime \prime}\right)_{12}=B_{12} \sigma\left(T_{h}^{4}-T_{c}^{4}\right)$ where $B_{12}$ is the Gebhart factor which solely depends on (i) the radiative properties of the wall and (ii) the view factors with any surface element involved in the cavity. These view factors remain constants when $L$ varies because all the aspect ratios between geometric elements are preserved. And so is the $B_{12}$ factor. Moreover, the temperature between the two active walls being weak, the $\left(T_{h}^{4}-T_{c}^{4}\right)$ term may be linearized around the central temperature $T_{0}$ (which is kept constant whatever $L$ ). As a result, the flux may be expressed as $\left(q_{r}^{\prime \prime}{ }^{\prime \prime}\right)_{12}=h_{r}\left(T_{h}-T_{c}\right)$, where the $h_{r}$ coefficient is expected to be independent of the cavity size. This is what is actually observed in table 5 , as well as a linear increase of $N u^{r}$ with $L$ (since $N u^{r} \propto h_{r} L$ ). Small variations of $h_{r}$ with length in case A may be explained by the choice of using the same radiative mesh whatever the cavity length. However, this choice seems reasonable as the difference does not exceed $0.2 \%$ between $L=1 \mathrm{~m}$ and $L=3 \mathrm{~m}$, which will ensure to study the effect of gas and/or wall radiation. In case C, the maximum error is less than $0.1 \%$. In case $\mathrm{B}, h_{r}$ decreases slightly $(\approx 1.5 \%)$ due to absorption by the gas that increase with optical path. The decrease is enhanced $(\approx 2.7 \%)$ by wall radiation (case $\mathrm{D})$.

Modification of passive walls temperature distribution also affects radiative exchange with gas and so the radiative source term distribution defined as $S_{r}^{*}=-\nabla \cdot \mathbf{q}_{\mathrm{r}} L^{2} /(\lambda \Delta T \sqrt{R a})$. To explain the differences in the internal structures of flows in case $\mathrm{B}$ and $\mathrm{D}$, it is convenient to compare the spatial distributions of the non-dimensional radiative source term in the midplane $y^{*}=0.5$ (Fig. 13). In both cases, the cavity length tends to decrease the horizontal gradient of the radiative source in the core region. In other words, the effects of passive walls decrease with length as iso- $S_{r}^{*}$ are straightened vertically.

Fig. 14 compares the evolution of the radiative source term along the lines $\left(x^{*}, 0.9,0.9\right)$ and $\left(1-x^{*}, 0.1,0.1\right)$ in order to check for centrosymmetry. In both cases (B and D), the superposition of radiative heat source profiles indicates that the centrosymmetry of the problem is conserved even though radiation is known to be strongly non-linear. It is worth pointing out that in this configuration, radiation is intense enough to alter the flow pattern but not enough to introduce significant non-linear effects. This observation is only valid for the temperature differences and cavity dimensions considered in the present study. Each of the source terms in the system of equations (1-5) (e.g. radiative source term, Boussinesq buoyancy term) being centrosymmetric, so is the fluid flow structure.

\section{4. $3 D$ effects}

3D effects are fairly well exhibited by plotting two opposed isosurfaces of the y-component of the velocity $v^{*}$ for cases $\mathrm{B}$ and $\mathrm{D}$, for the three cavity lengths studied and at $R a=10^{6}$ (Fig. 15). As shown in Table 3, the cavity length tends to increase the lateral velocity $v^{*}$, especially in case B. For $L=1 m$, relatively moderate transverse velocities are observed in the lower part of the hot wall and in the upper part of the cold wall (centrosymmetry). When increasing $L$, the transverse flow involves nearly the whole height of the wall. Two important cells appear, for $L=2 m$ on top and bottom walls. Then for $L=3 m$, each of these cells is divided into two (in case B) and three (in case D) cells whose directions alternate. In both cases, the lateral velocity remains important close to vertical lateral walls. While considering the number and shape of the cells, the flow becomes more complex when both gas and wall radiation are involved (case D). As the cavity size increases, 
the flow increases in the center of the cavity which results in an intensification of the rotating core flow as revealed by the path-lines in Fig. 15.

\section{Conclusions}

Three-dimensional coupled simulations of a natural convection flow combined to surface and molecular gas radiation have been performed in a cubical differentially heated cavity filled with an air $/ \mathrm{H}_{2} \mathrm{O}$ mixture at $\mathrm{Ra}=10^{6}$. The radiative source terms have been calculated using a 3D Discrete Ordinate Method and coupled to a finite volume scheme to solve the mass, momentum, and energy equations within the flow. The spectral dependency of the gas absorption has been taken into account using the SLW model through the calculation of the absorption coefficient of a certain number of fictitious gray gases. The model was first validated against benchmark solutions. Then, three radiative configurations are considered plus a pure convective reference case in order to study the effect of the cavity size on the flow and heat transfer characteristics. Three cavity lengths of 1,2 and 3m were used along with a particular gas mixture with small amounts of $\mathrm{H}_{2} \mathrm{O}$ at room temperature and atmospheric pressure in order to mimic situations that may be encountered in housing.

For the participating medium cases, the results show a strong dependency of temperature and velocity fields to cavity length, whatever the radiative properties of the adiabatic wall. We observe an increase of the global circulation in the cavity and the disappearance of the stagnant core region, resulting mostly from gas radiation effects. These effects are amplified when both insulated wall and gas radiation exist.

In participating medium with reflecting insulated walls, we underline a decrease of the stratification parameter with cavity length, whereas no significant effect is observed in the radiating insulated walls case. In this case, the change of scale does not modify the adiabatic wall temperature distribution, especially the top and bottom ones.

Whatever the nature of the adiabatic walls, the radiative exchanges between the two isothermal walls decrease slightly with cavity length as the fraction of radiation absorbed by the medium increases with optical length. On the other hand, the convective heat transfer tends to increase although the intensity of the transfer is strongly dependent of the flow pattern, especially the shape of the horizontal jets. While the jets are relatively flat for low values of the cavity length, they tend to thicken and split up making the convective exchange mapping more complex. Wall radiation accentuates these effects.

The cavity length was shown to magnify the 3D effects, observed here by means of the velocity in the transverse direction (y). Wall radiation enhances the radiative effects due to gas and a more complex flow appears. It is worth pointing out that centosymmetry of the dynamic and thermal fields are preserved since only weak temperature differences are prescribed in this study.

The present work is an attempt to understand the effect of real-gas radiation for the prediction of heat transfer coupled with fluid dynamic. Observation made may also be useful for the understanding of the influence of radiation, through radiative properties of the medium and the length of the container, in turbulent flows which remains a challenging issue.

\section{References}

[1] G. De Vahl Davis and I. P. Jones. Natural convection in a square cavity: A comparison exercise. International Journal for Numerical Methods in Fluids, 3(3):227248, May 1983. ISSN 0271-2091, 1097-0363. doi: 10.1002/fld.1650030304. URL http://doi.wiley.com/10.1002/fld.1650030304. 
[2] P. Le Quéré. Accurate solutions to the square thermally driven cavity at high rayleigh number. Computers \& Fluids, 20(1):29-41, January 1991. ISSN 00457930. doi: 10.1016/0045-7930(91)90025-D. URL http://linkinghub.elsevier.com/retrieve/pii/004579309190025D.

[3] D. W. Larson and R. Viskanta. Transient combined laminar free convection and radiation in a rectangular enclosure. Journal of Fluid Mechanics, 78(01):65, November 1976. ISSN 0022-1120, 1469-7645. doi: 10.1017/S0022112076002334. URL http://www . journals . cambridge. org/abstract_S0022112076002334.

[4] H.F. Nouanegue, A. Muftuoglu, and E. Bilgen. Heat transfer by natural convection, conduction and radiation in an inclined square enclosure bounded with a solid wall. International Journal of Thermal Sciences, 48(5):871-880, May 2009. ISSN 12900729. doi: 10.1016/j.ijthermalsci.2008.06.008. URL http://linkinghub.elsevier.com/retrieve/pii/S1290072908001452.

[5] H. Sun, E. Chénier, and G. Lauriat. Effect of surface radiation on the breakdown of steady natural convection flows in a square, air-filled cavity containing a centered inner body. Applied Thermal Engineering, 31(6-7):1252-1262, May 2011. ISSN 13594311. doi: 10.1016/j.applthermaleng.2010.12.028. URL http://linkinghub.elsevier.com/retrieve/pii/S1359431110005442.

[6] G. Lauriat. Combined radiation-convection in gray fluids enclosed in vertical cavities. Journal of Heat Transfer, 104(4):609, 1982. ISSN 00221481. doi: 10.1115/1.3245175. URL http://HeatTransfer . asmedigitalcollection. asme.org/article. aspx?articleid=1437795.

[7] A. Yucel, S. Acharya, and M. L. Williams. Natural convection and radiation in a square enclosure. Numerical Heat Transfer, Part A: Applications, 15(2):261-278, March 1989. ISSN 1040-7782, 1521-0634. doi: 10.1080/10407788908944688. URL http://www.tandfonline.com/doi/abs/10.1080/10407788908944688.

[8] K. Lari, M. Baneshi, S. A. Gandjalikhan Nassab, A. Komiya, and S. Maruyama. Combined heat transfer of radiation and natural convection in a square cavity containing participating gases. International Journal of Heat and Mass Transfer, 54(23-24):5087-5099, November 2011. ISSN 00179310. doi: 10.1016/j.ijheatmasstransfer.2011.07.026. URL http://linkinghub.elsevier.com/retrieve/pii/S0017931011004029.

[9] G. Colomer, R. Cònsul, and A. Oliva. Coupled radiation and natural convection: Different approaches of the slw model for a non-gray gas mixture. Journal of Quantitative Spectroscopy and Radiative Transfer, 107(1):3046, September 2007. ISSN 00224073. doi: 10.1016/j.jqsrt.2006.12.011. URL http://linkinghub.elsevier.com/retrieve/pii/S0022407307000064.

[10] M. N. Borjini, H. Ben Aissia, K. Halouani, and B. Zeghmati. Effect of radiative heat transfer on the three-dimensional boyancy flow in cubic enclosure heated from the side. International Journal of Heat and Fluid Flow, 29(1):107-118, February 2008. ISSN 0142727X. doi: 10.1016/j.ijheatfluidflow.2007.07.012. URL http://linkinghub.elsevier.com/retrieve/pii/S0142727X07001178.

[11] P. Kumar and V. Eswaran. A numerical simulation of combined radiation and natural convection in a differential heated cubic cavity. Journal of Heat Trans- 
fer, 132(2):023501, 2010. ISSN 00221481. doi: 10.1115/1.4000180. URL http://HeatTransfer. asmedigitalcollection . asme.org/article. aspx?articleid=1449835.

[12] V. Borget, F. Bdéoui, A. Soufiani, and P. Le Quéré. The transverse instability in a differentially heated vertical cavity filled with molecular radiating gases. i. linear stability analysis. Physics of Fluids, 13(5):1492, 2001. ISSN 10706631. doi: 10.1063/1.1358309. URL http://scitation.aip.org/content/aip/journal/pof2/13/5/10.1063/1.1358309.

[13] A. Ibrahim, D. Saury, and D. Lemonnier. Coupling of turbulent natural convection with radiation in an air-filled differentially-heated cavity at $R a=1.5 \times 10^{9}$. Computers \& Fluids, 88:115-125, December 2013. ISSN 00457930. doi: 10.1016/j.complluid.2013.09.006. URL http://linkinghub.elsevier.com/retrieve/pii/S0045793013003423.

[14] S. Laouar-Meftah, M. Cherifi, D. Lemonnier, and A. Benbrik. Gas radiation effects on opposing double-diffusive convection in a non-gray airh2o mixture. International Journal of Thermal Sciences, 77:38-46, March 2014. ISSN 12900729. doi: 10.1016/j.ijthermalsci.2013.10.004. URL http://linkinghub.elsevier.com/retrieve/pii/S1290072913002408.

[15] L. Soucasse, Ph. Rivire, and A. Soufiani. Natural convection in a differentially heated cubical cavity under the effects of wall and molecular gas radiation at rayleigh numbers up to 3 109. International Journal of Heat and Fluid Flow, 61, Part B:510 - 530, 2016. ISSN 0142-727X. doi: http://dx.doi.org/10.1016/j.ijheatfluidflow.2016.06.012. URL http://www. sciencedirect.com/science/article/pii/S0142727X16303186.

[16] Takuma Kogawa, Junnosuke Okajima, Astushi Sakurai, Atsuki Komiya, and Shigenao Maruyama. Influence of radiation effect on turbulent natural convection in cubic cavity at normal temperature atmospheric gas. International Journal of Heat and Mass Transfer, 104:456 - 466, 2017. ISSN 0017-9310. doi: https://doi.org/10.1016/j.ijheatmasstransfer.2016.08.059. URL http://www.sciencedirect.com/science/article/pii/S0017931016313370.

[17] S. Labrosse, J.-P. Poirier, and J.-L. Le Mouël. On cooling of the earth's core. Physics of the Earth and Planetary Interiors, 99(1-2):1-17, January 1997. ISSN 00319201. doi: 10.1016/S0031-9201(96)03207-4. URL http://linkinghub.elsevier.com/retrieve/pii/S0031920196032074.

[18] B. Gebhart. Buoyancy-Induced Flows And Transport. Taylor \& Francis, 1988. ISBN 9780891167280. URL https://books.google.fr/books?id=cXFiQgAACAAJ.

[19] M. K. Denison and B. W. Webb. A spectral line-based weighted-sumof-gray-gases model for arbitrary RTE solvers. Journal of Heat Transfer, 115(4):1004, 1993. ISSN 00221481. doi: 10.1115/1.2911354. URL http://HeatTransfer. asmedigitalcollection . asme.org/article. aspx?articleid=1441518.

[20] M. K. Denison and B. W. Webb. An absorption-line blackbody distribution function for efficient calculation of total gas radiative transfer. Journal of Quantitative Spectroscopy and Radiative Transfer, 50(5):499-510, November 1993. ISSN 00224073. doi: 10.1016/0022-4073(93)90043-H. URL http://linkinghub.elsevier.com/retrieve/pii/002240739390043H.

[21] M. K. Denison and B. W. Webb. Development and application of an absorptionline blackbody distribution function for CO2. International Journal of Heat and Mass Transfer, 38 
(10):1813-1821, July 1995. ISSN 00179310. doi: 10.1016/0017-9310(94)00297-9. URL http://linkinghub.elsevier.com/retrieve/pii/0017931094002979.

[22] F. Archambeau, M. Namane, and M. Sakiz. Code saturne: A finite volume code for turbulent flows. International Journal On Finite Volumes, 2004.

[23] W. A. Fiveland. Discrete-ordinates solutions of the radiative transport equation for rectangular enclosures. Journal of Heat Transfer, 106(4):699, 1984. ISSN 00221481. doi: 10.1115/1.3246741. URL http://HeatTransfer.asmedigitalcollection. asme.org/article. aspx?articleid=1438452.

[24] L. Soucasse, P. Rivière, S. Xin, P. Le Quéré, and A. Soufiani. Numerical study of coupled molecular gas radiation and natural convection in a differentially heated cubical cavity. Computational Thermal Sciences, 4(4):335-350, 2012. ISSN 1940-2503. doi: 10.1615/ComputThermalScien.2012005118. URL http://www.dl . begellhouse.com/journals/648192910890cd0e, 2050cca31f3c9948, 3cb69dfb2be801

[25] L. Soucasse, P. Rivière, and A. Soufiani. Monte carlo methods for radiative transfer in quasi-isothermal participating media. Journal of Quantitative Spectroscopy and Radiative Transfer, 128:34-42, October 2013. ISSN 00224073. doi: 10.1016/j.jqsrt.2012.07.008. URL http://linkinghub.elsevier.com/retrieve/pii/S0022407312003330.

[26] F. Liu. Numerical solutions of three-dimensional non-grey gas radiative transfer using the statistical narrow-band model. Journal of Heat Transfer, 121(1):200, 1999. ISSN 00221481. doi: 10.1115/1.2825944. URL http: //HeatTransfer . asmedigitalcollection . asme.org/article . aspx?articleid=1443580.

[27] J. T. Pearson, B. W. Webb, V. P. Solovjov, and J. Ma. Updated correlation of the absorption line blackbody distribution function for $h_{2} O$ based on the HITEMP2010 database. Journal of Quantitative Spectroscopy and Radiative Transfer, 128:10-17, October 2013. ISSN 00224073. doi: 10.1016/j.jqsrt.2012.07.016. URL http://linkinghub.elsevier.com/retrieve/pii/S0022407312003500.

[28] S. Xin and P. Le Quéré. An extended chebyshev pseudo-spectral benchmark for the 8:1 differentially heated cavity. International Journal for Numerical Methods in Fluids, 40 (8):981-998, November 2002. ISSN 0271-2091, 1097-0363. doi: 10.1002/fld.399. URL http://doi.wiley.com/10.1002/fld.399. 


\section{Figures}
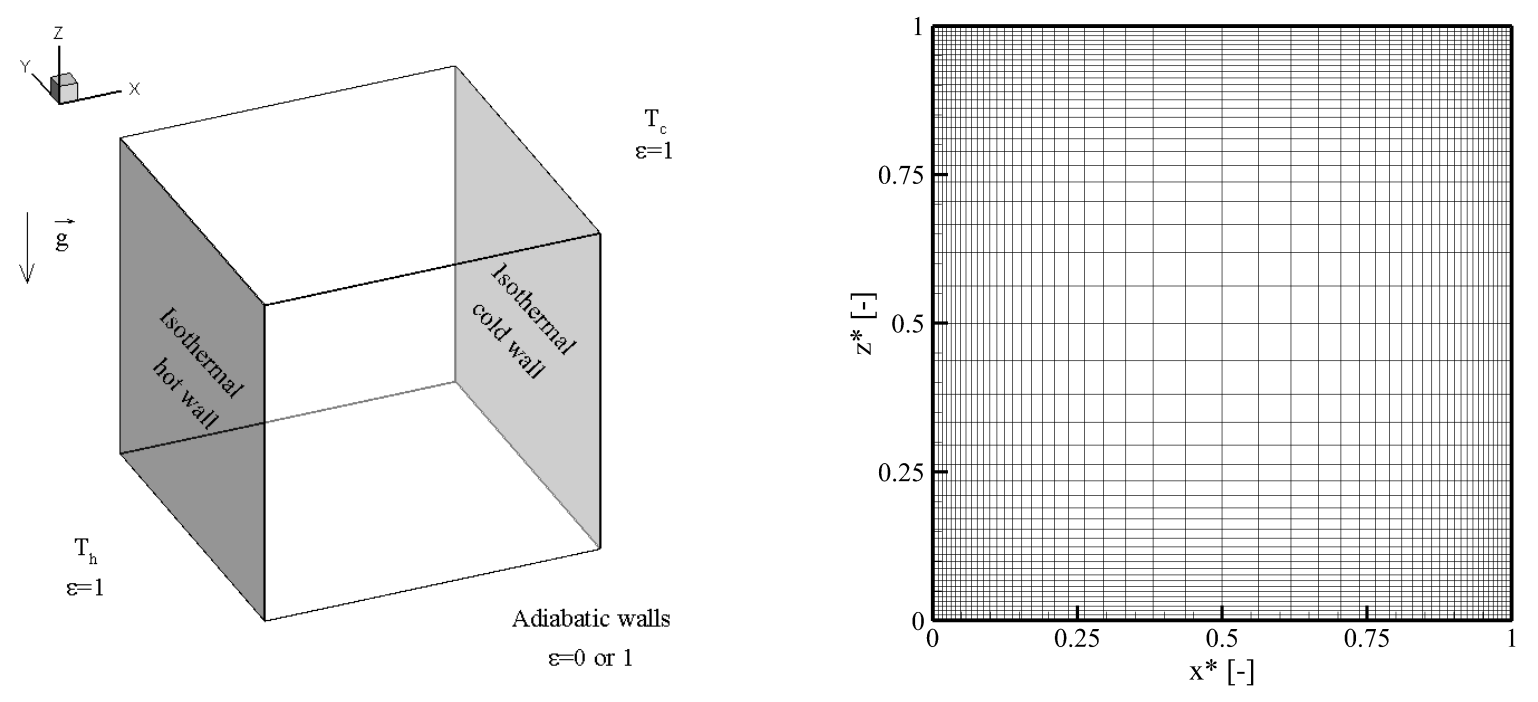

Figure 1: On left, three-dimensional differential heated cavity scheme and boundary conditions. On right, computational mesh in the y-plane. The node distribution follows a hyperbolic cosine law. 

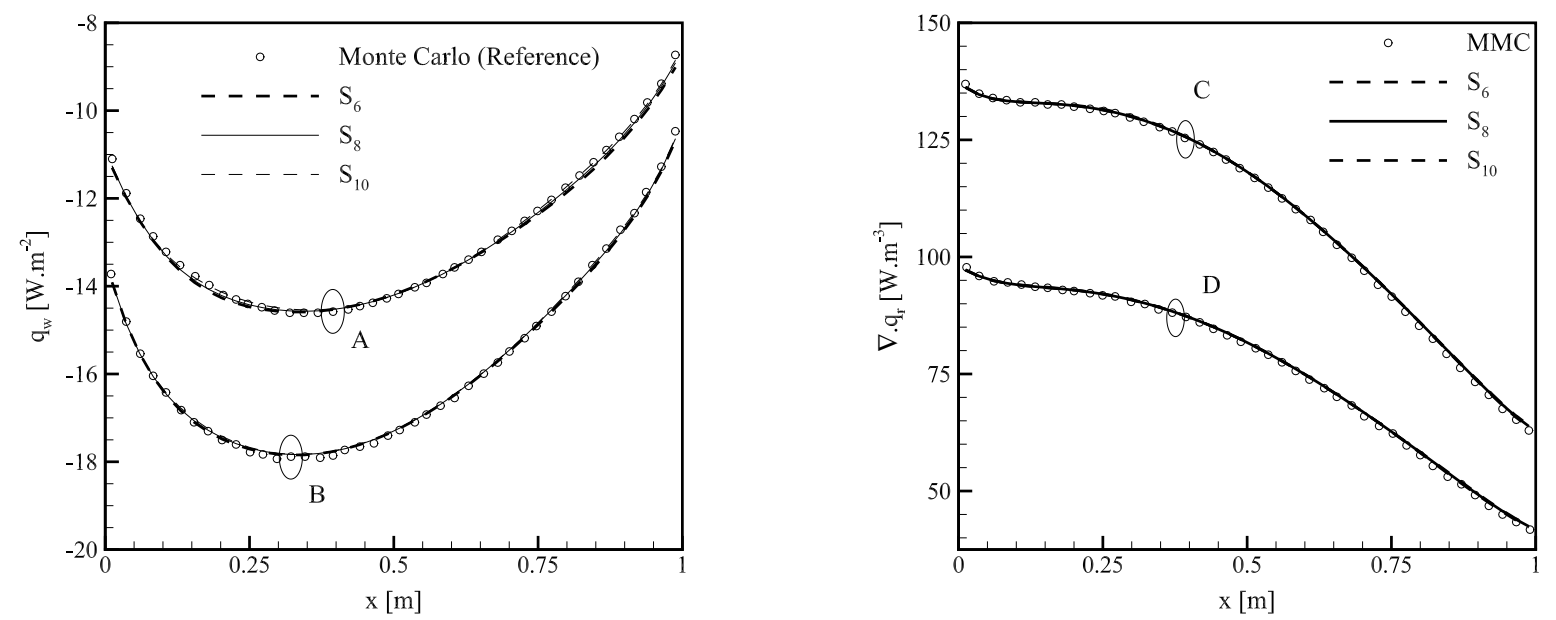

Figure 2: (a) Incoming radiative flux profile along the lines $\mathrm{A}(\mathrm{x}, 0.25 \mathrm{~m}, 1 \mathrm{~m})$ and $\mathrm{B}(\mathrm{x}, 0.25 \mathrm{~m}, 0 \mathrm{~m})$. (b) Radiative volumetric power along $\mathrm{C}(\mathrm{x}, 0.25 \mathrm{~m}, 0.25 \mathrm{~m})$ and $\mathrm{D}(\mathrm{x}, 0.25 \mathrm{~m}, 0.75 \mathrm{~m})$. Comparisons between DOM predictions for different angular quadrature sets and MMC predictions [24] 

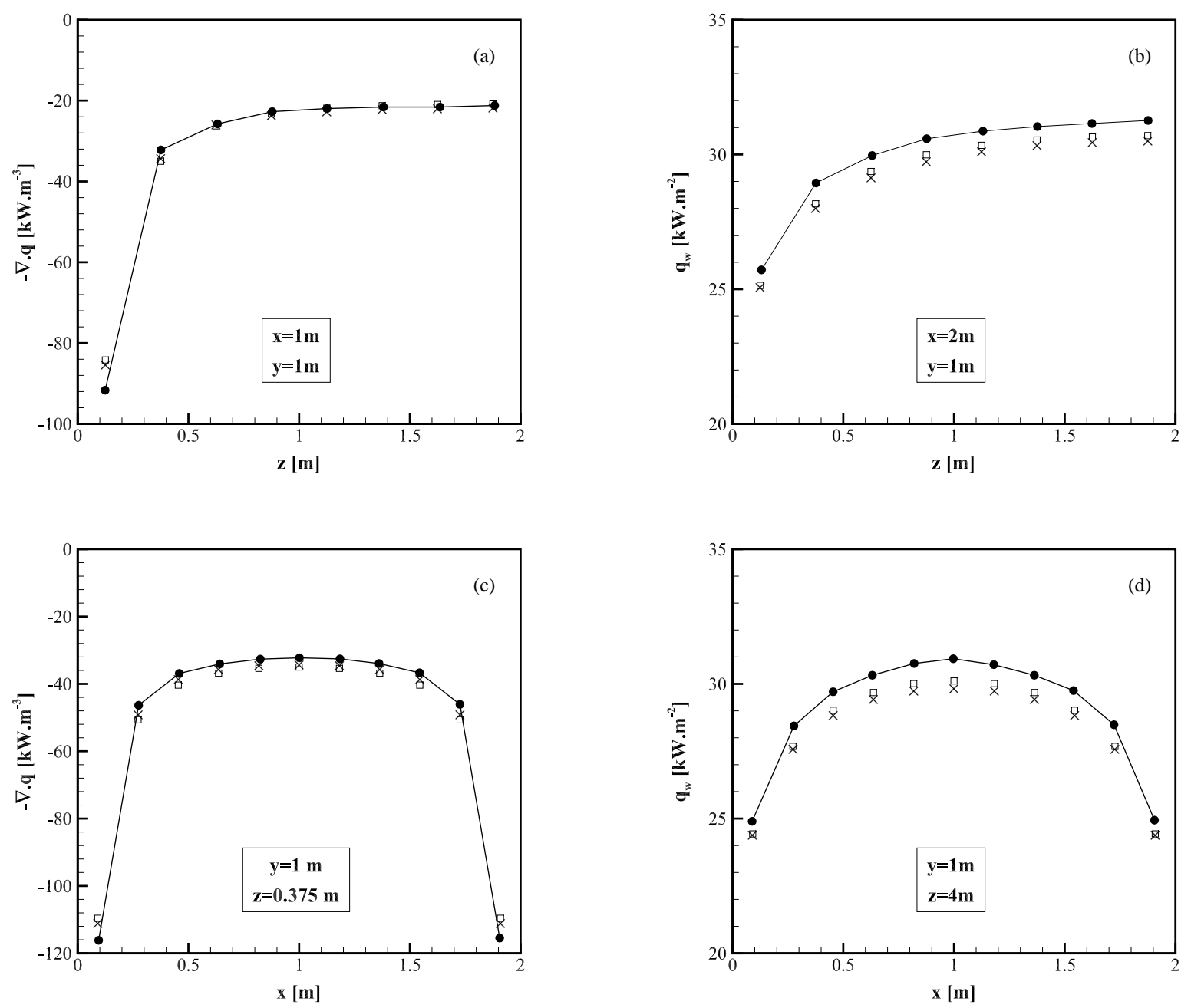

Figure 3: Distributions of radiative heat source (a) and (c) and incident heat flux (b) and (d). Comparison of DOM results when coupled with the SLW model using $8(\times)$ and $20(\square)$ cross sections against calculations based on ray-tracing and the SNB model $(\bullet)[26]$. 

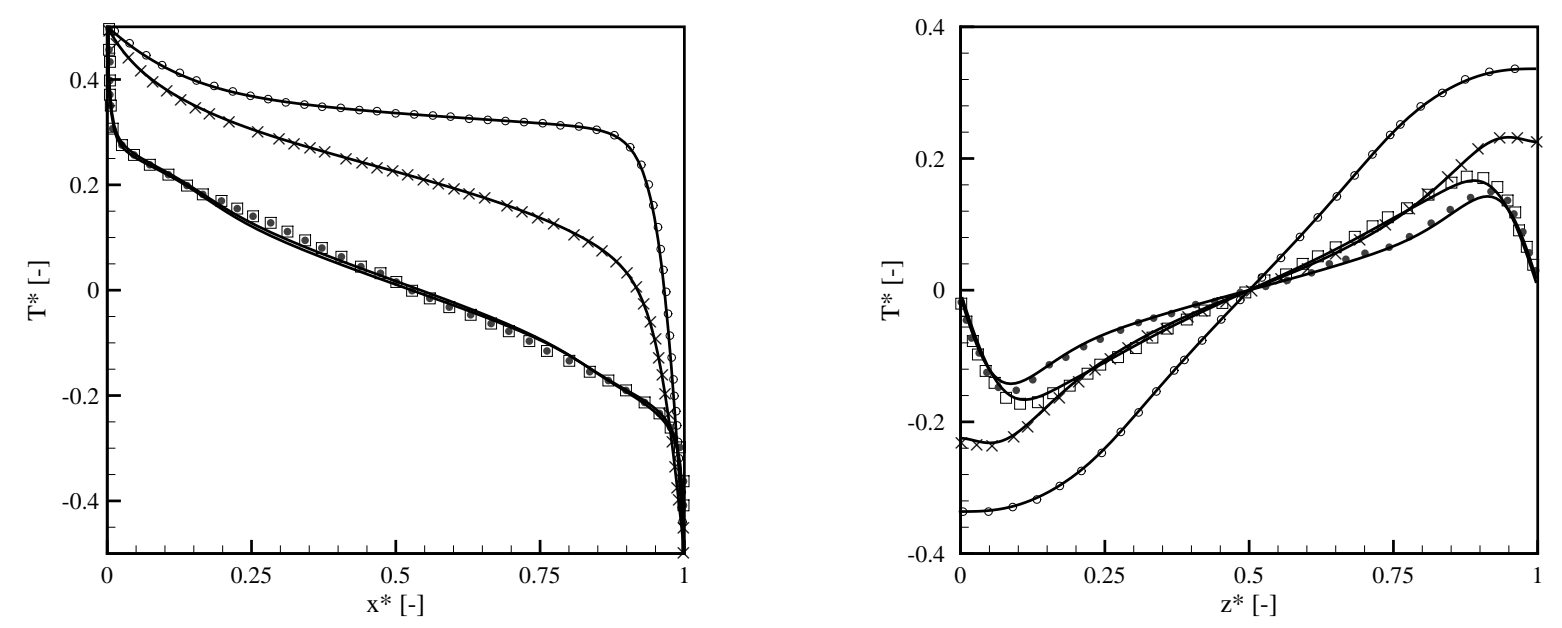

Figure 4: Left, temperature profile at the top wall $\left(x^{*}, \overline{y^{*}}, 1\right)$. Right, vertical temperature profile $\left(0.5, \overline{y^{*}}, z^{*}\right)$. Results are averaged over y and $R a=10^{6}$. Solid lines correspond to present work, symbols to references by Soucasse et al. $[24]$. Case A (o), B (×) C $(\square)$ and D $(\bullet)$. 

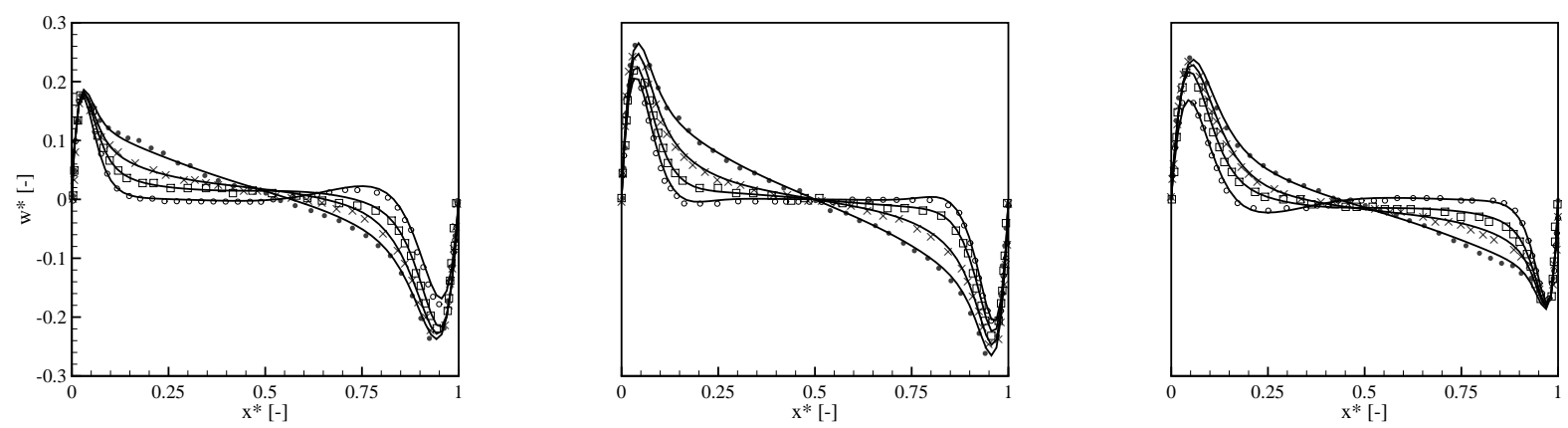

Figure 5: Vertical velocity profile at three different heights. Left: $\left(x^{*}, \overline{y^{*}}, 0.25\right)$. Center: $\left(x^{*}, \overline{y^{*}}, 0.5\right)$. Right: $\left(x^{*}, \overline{y^{*}}, 0.75\right)$. Results are averaged over $\mathrm{y}$ and $R a=10^{6}$. Solid lines correspond to present work, symbols to references by Soucasse et al. [24]. Case A (o), B $(\times)$ C $(\square)$ and D $(\bullet)$. 


$$
L=1 m
$$
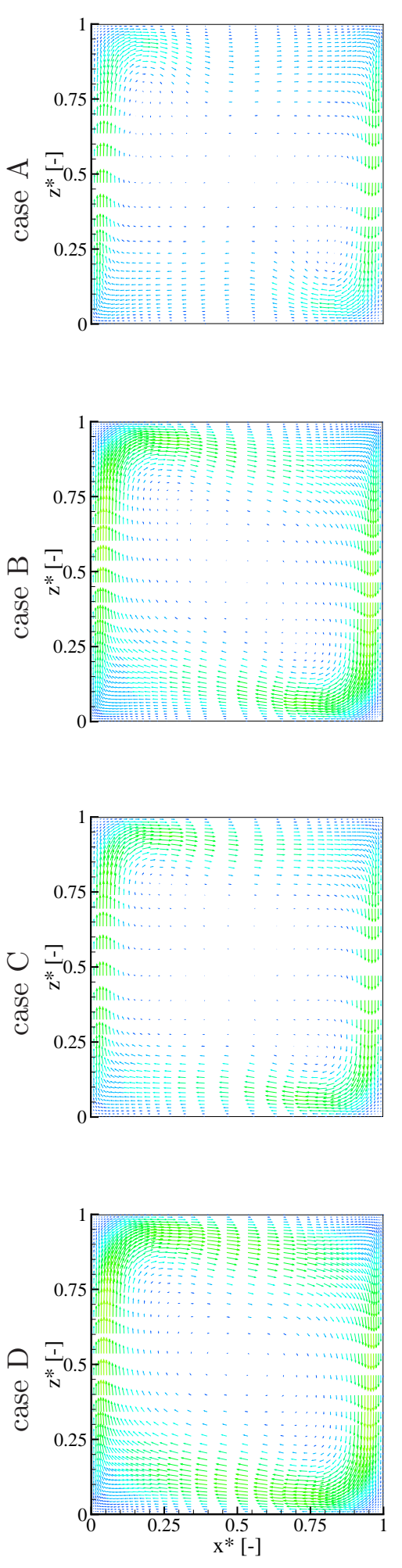

$\mathrm{U} *[-]$
$L=2 m$
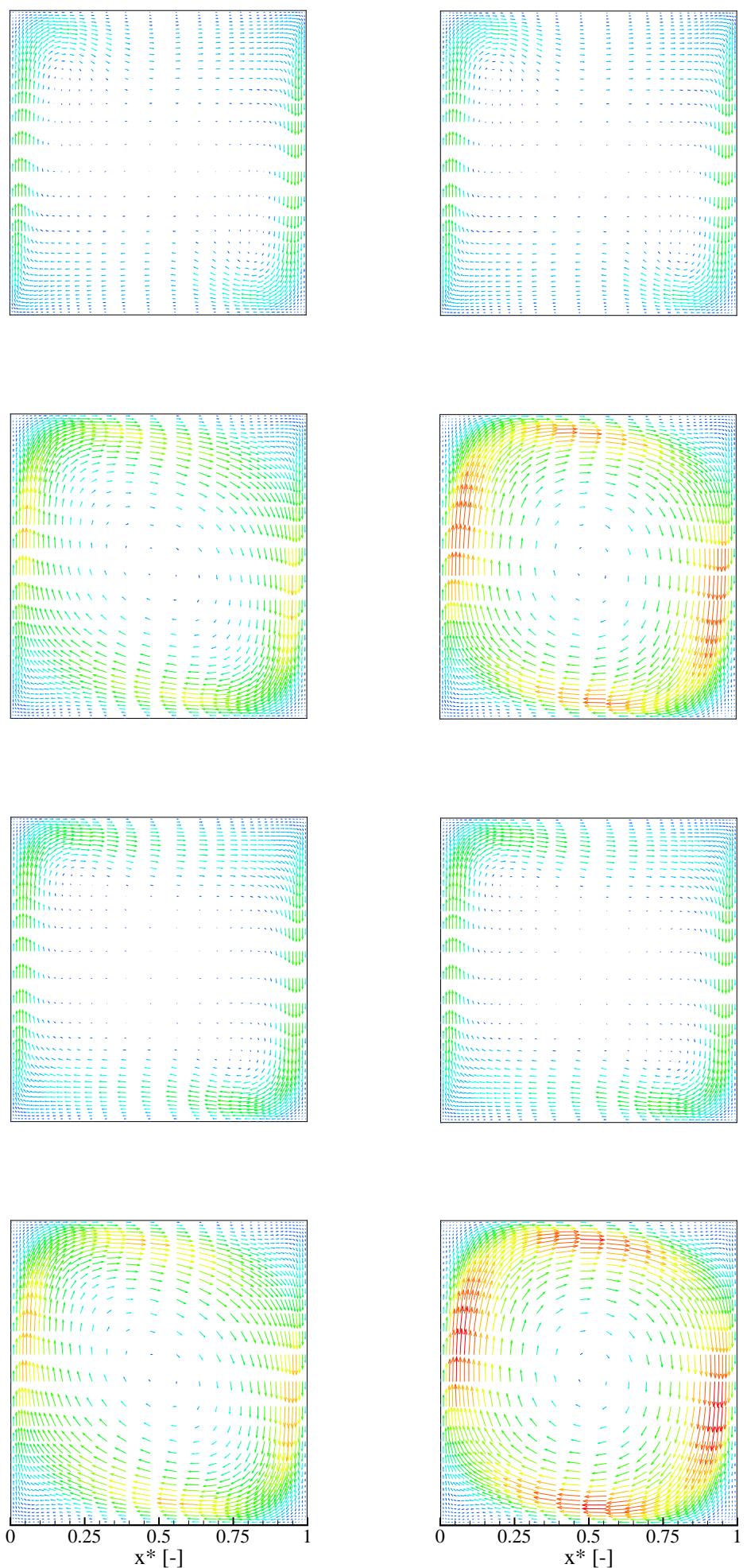

Figure 6: Flow structure description in the vertical mid-plane $y^{*}=0.5$ for $R a=10^{6}$. Velocity vectors are colored by intensity 

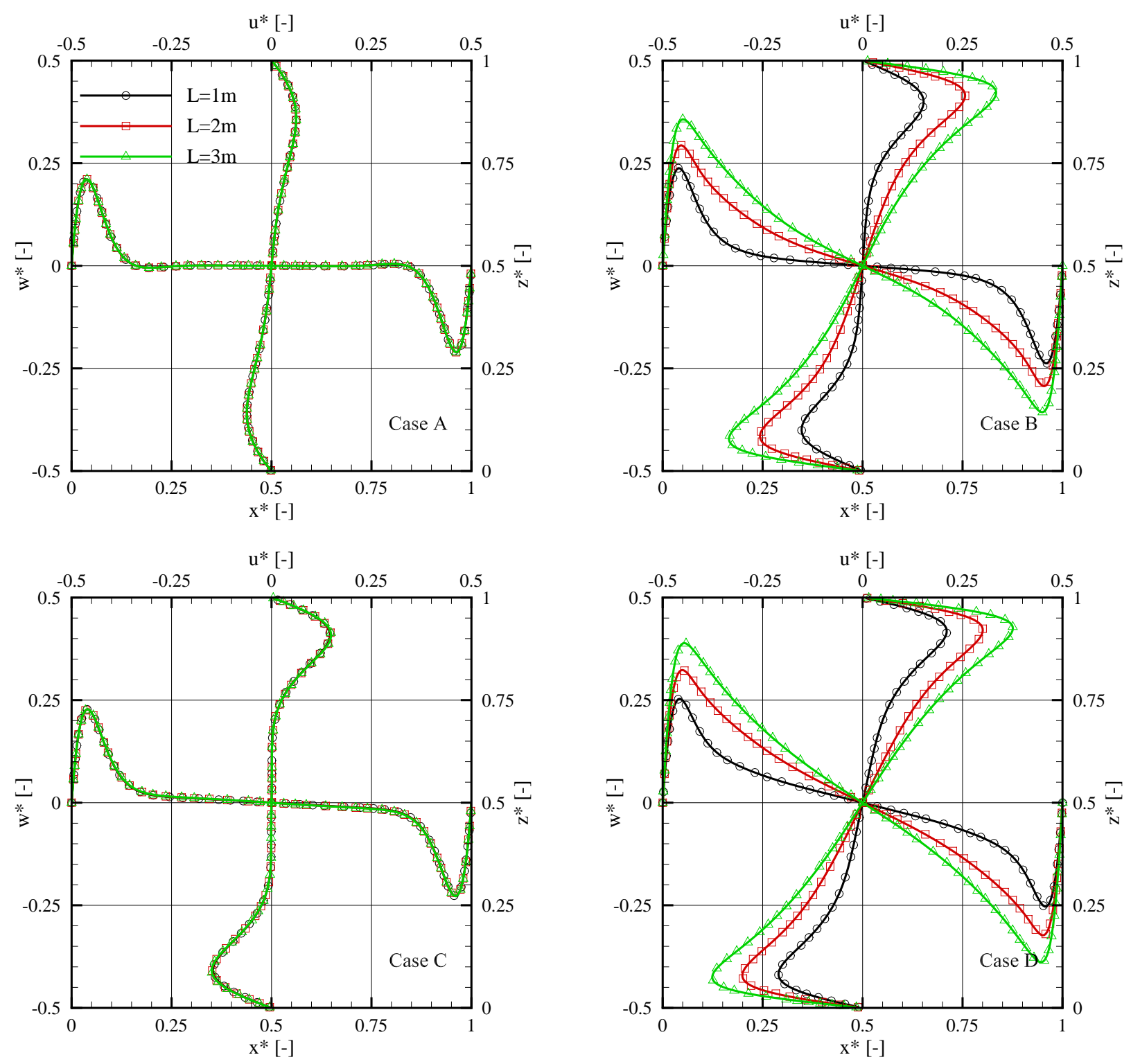

Figure 7: Vertical and horizontal velocity at $x^{*}=z^{*}=0.5$ (data are averaged over y) for $R a=10^{6}$ and different cavity lengths: $L=1 m(\circ), L=2 m(\square)$ and $L=3 m(\Delta)$. 
Case A

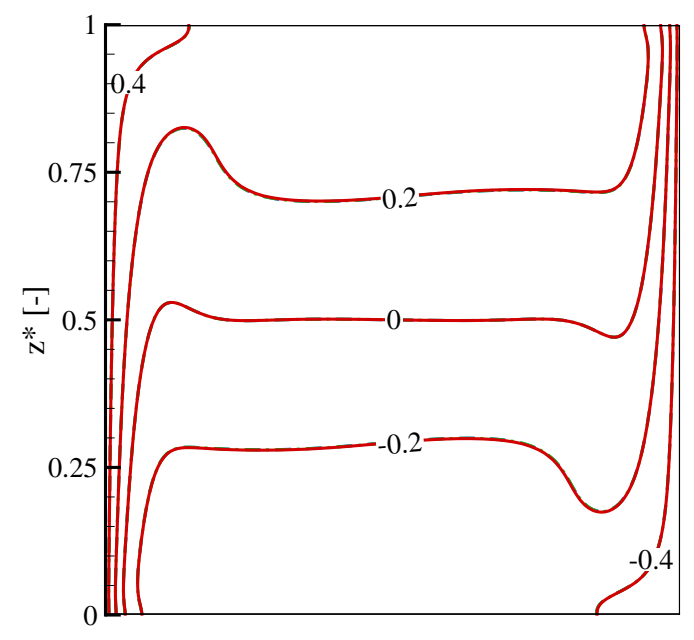

Case C

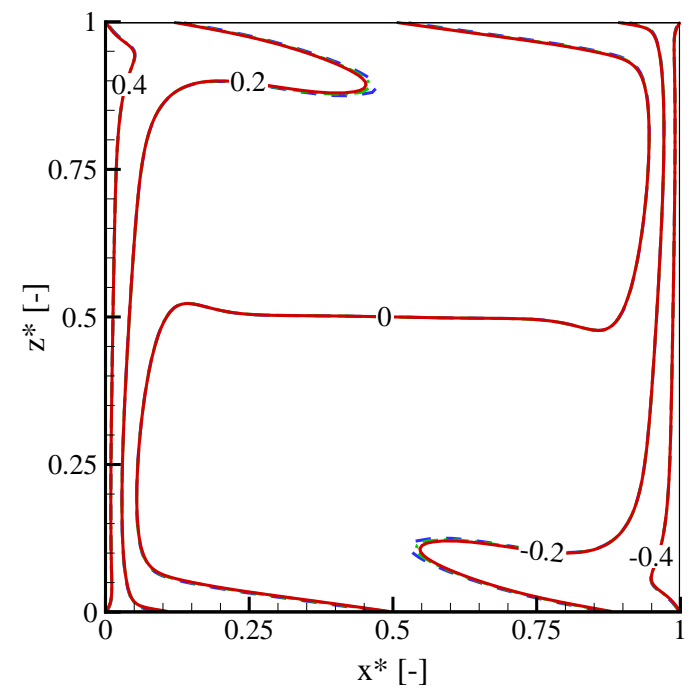

Case B

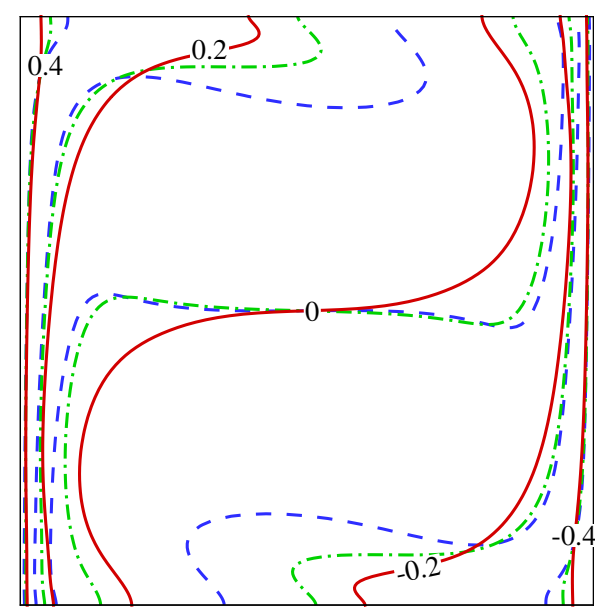

Case D

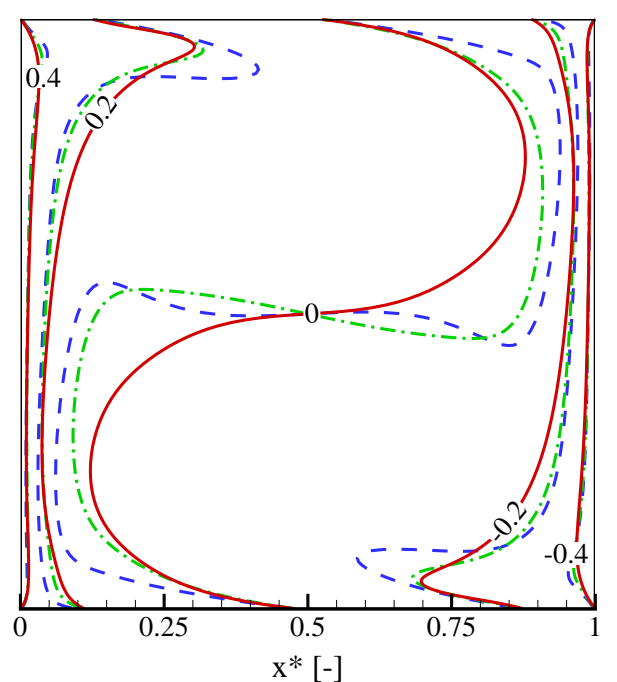

Figure 8: Iso-values $\left(T^{*} \in[-0.4 ;-0.2 ; 0 ; 0.2 ; 0.4]\right)$ of the mean temperature in the plane $y^{*}=0.5$ for the different cases at $R a=10^{6}$ and $L=1 \mathrm{~m}$ (dashed line), $L=2 \mathrm{~m}$ (dash-dot line) and $L=3 \mathrm{~m}$ (solid line). 

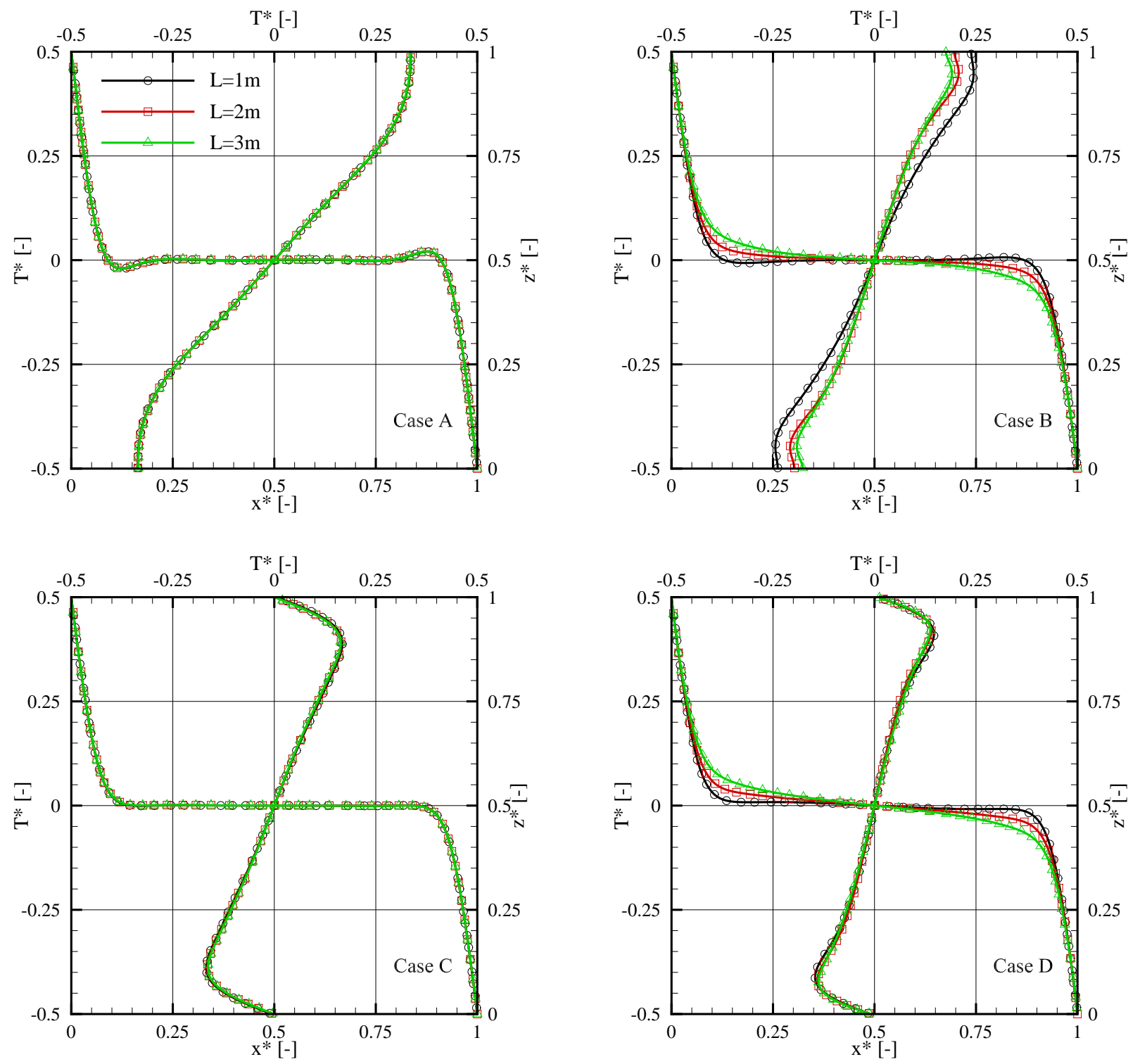

Figure 9: Temperature profiles at $x^{*}=z^{*}=0.5$ (data are averaged over $y^{*}$ ) for $R a=10^{6}$ and different cavity lengths: $L=1 m(\circ), L=2 m(\square)$ and $L=3 m(\Delta)$. 

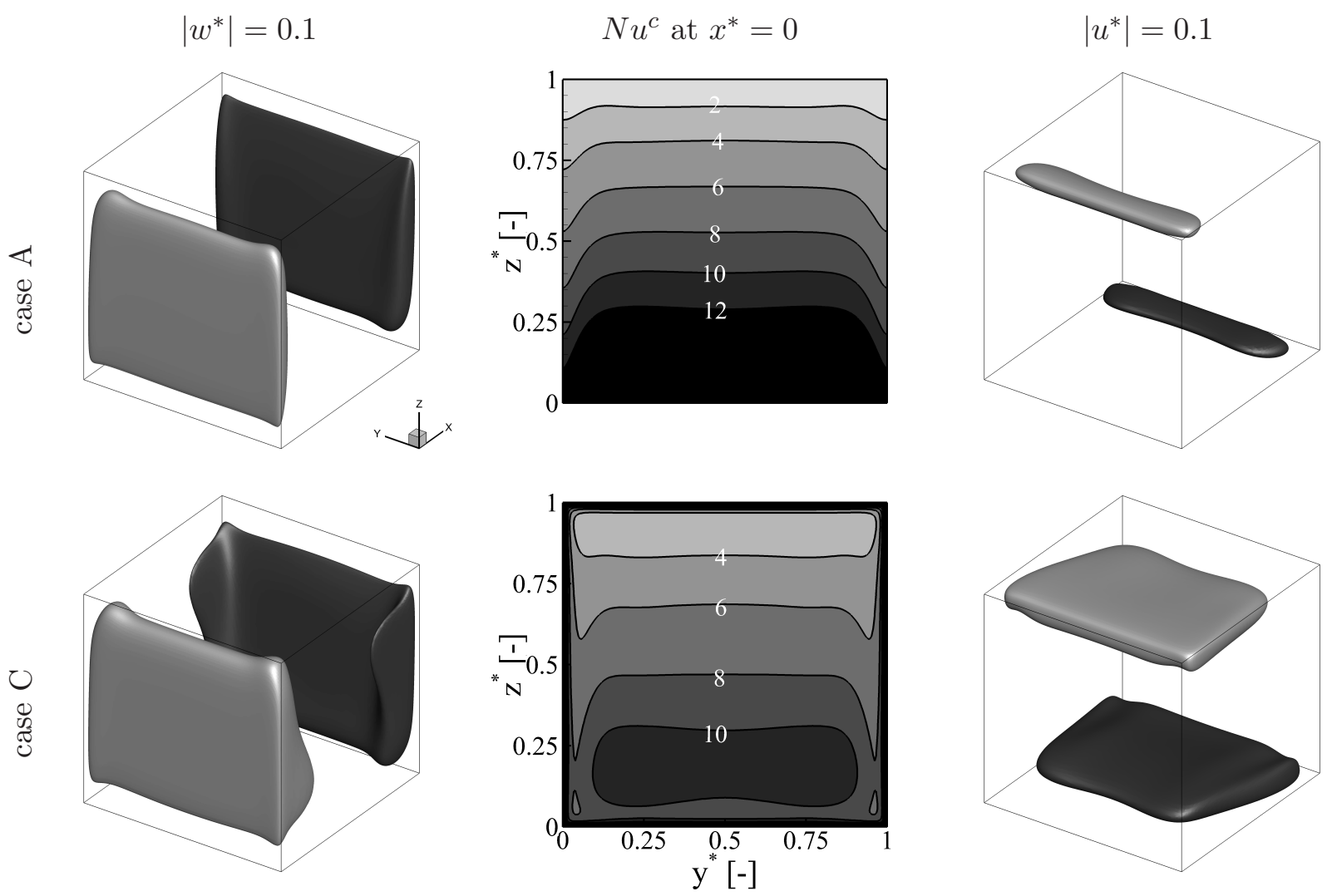

Figure 10: Effect of wall radiation by comparison between the transparent cases (A and C) at $R a=10^{6}$. On the left, vertical velocity $w^{*}=0.1$ (light-grey) and $w^{*}=-0.1$ (dark-grey). In the center, $2 \mathrm{D}$ convective Nusselt on the hot wall $\left(x^{*}=0\right)$. On the right, horizontal velocity $u^{*}=0.1$ (light-grey) and $u^{*}=-0.1$.(dark-grey). 

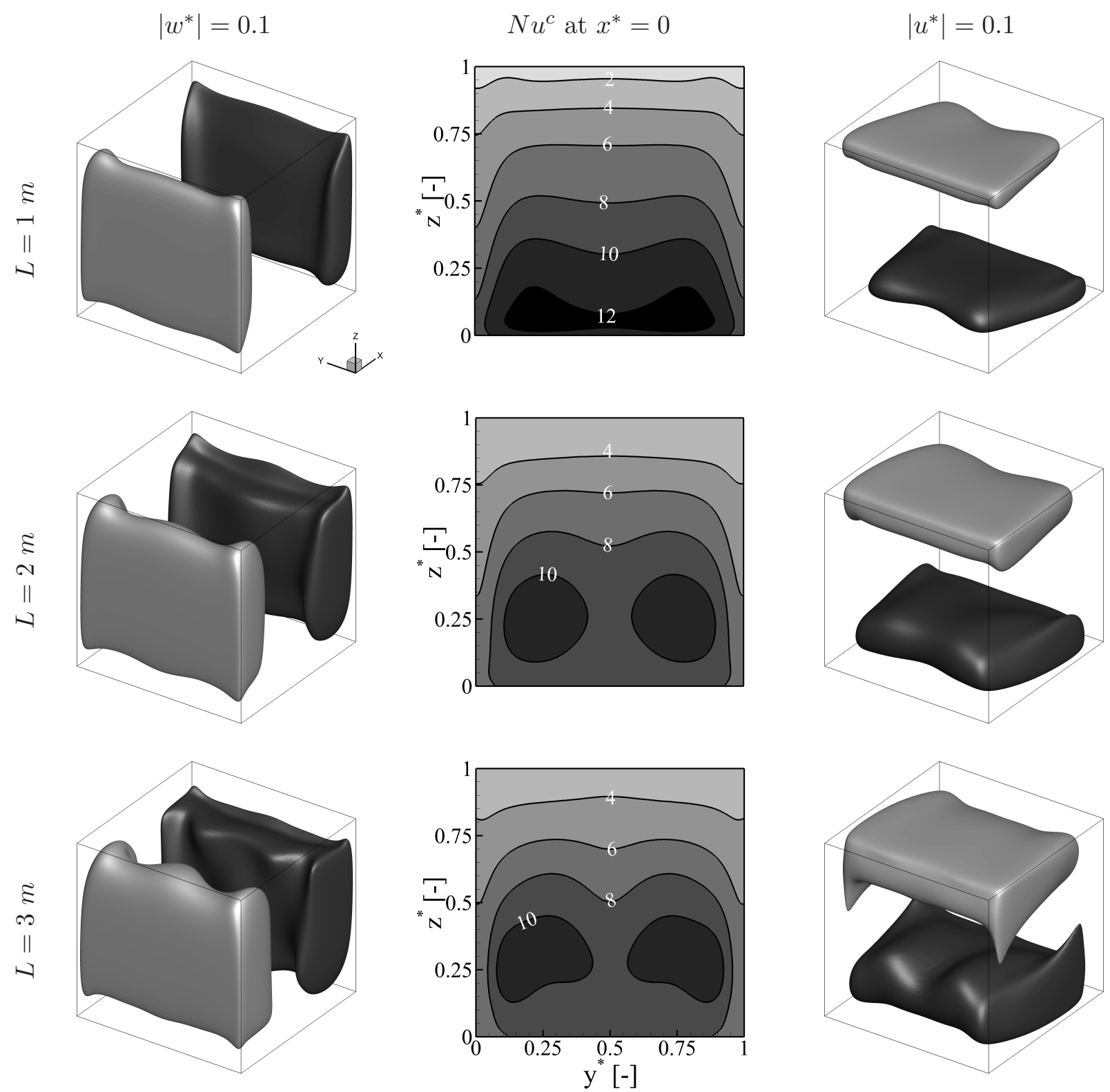

Figure 11: Effect of cavity length with purely reflective adiabatic walls (case B) at $R a=10^{6}$. On the left, vertical velocity $w^{*}=0.1$ (light-grey) and $w^{*}=-0.1$ (dark-grey). In the center, 2D convective Nusselt on the hot wall $\left(x^{*}=0\right)$. On the right, horizontal velocity $u^{*}=0.1$ (light-grey) and $u^{*}=-0.1$.(dark-grey). 

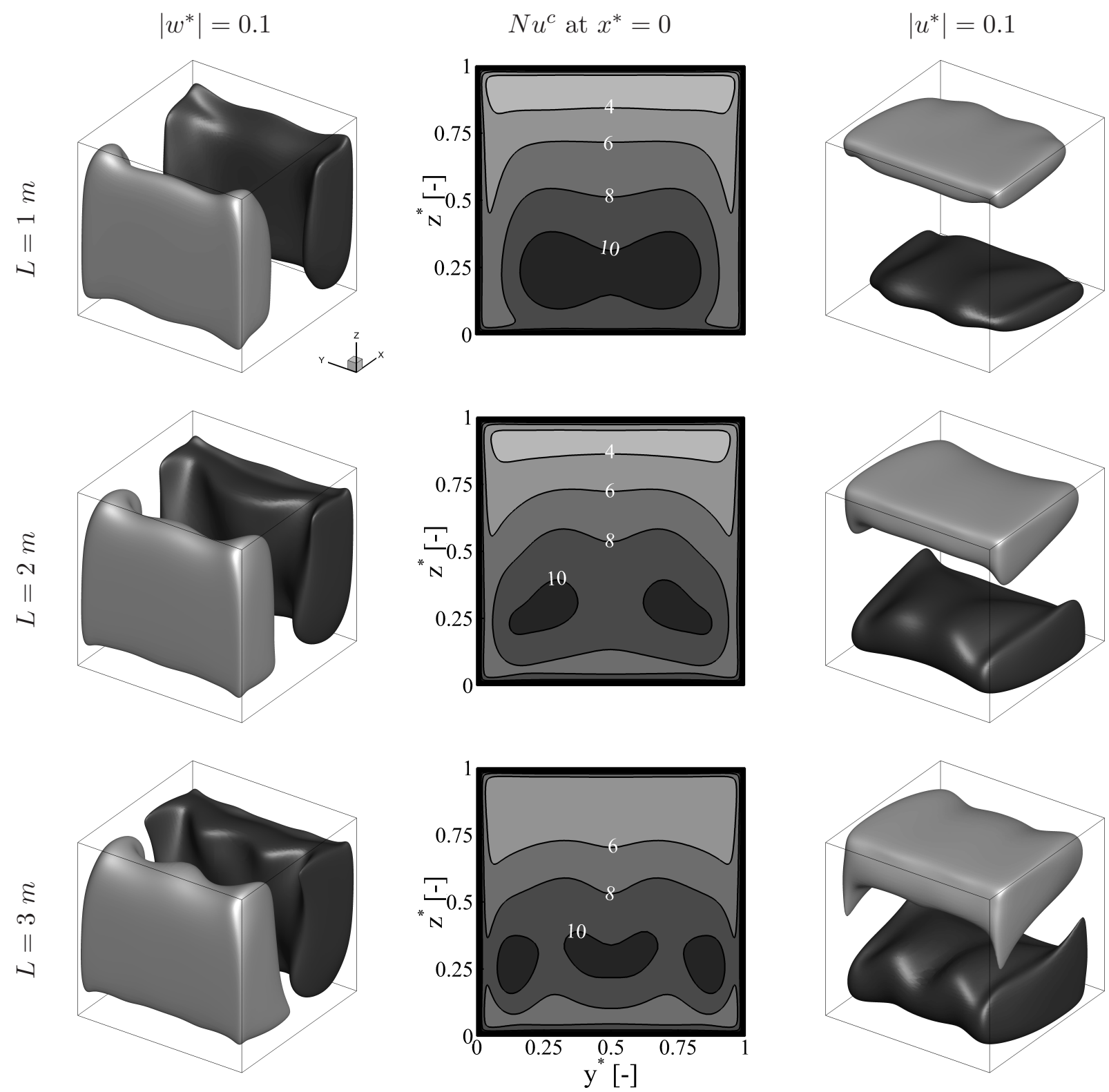

Figure 12: Effect of cavity length with purely absorbing adiabatic walls (case D) at $R a=10^{6}$. On the left, vertical velocity $w^{*}=0.1$ (light-grey) and $w^{*}=-0.1$ (dark-grey). In the center, 2D convective Nusselt on the hot wall $\left(x^{*}=0\right)$. On the right, horizontal velocity $u^{*}=0.1$ (light-grey) and $u^{*}=-0.1$ (dark-grey). 
Case B

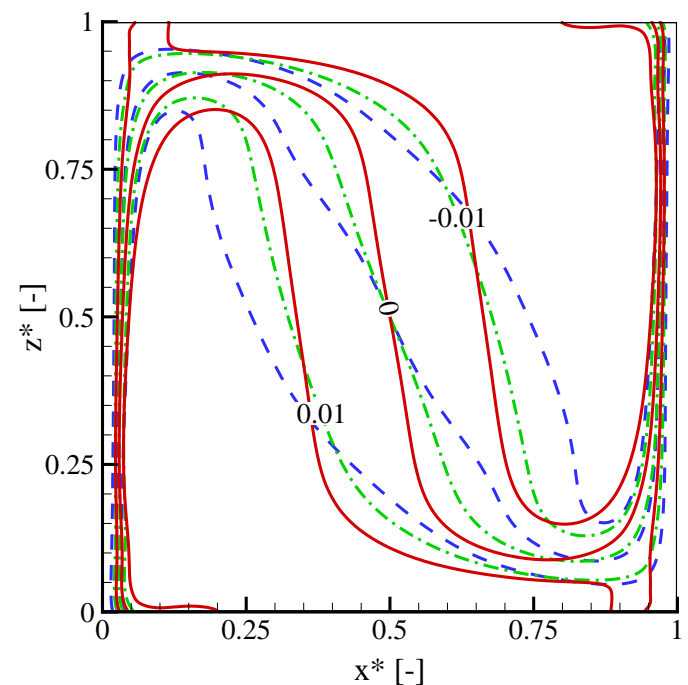

Case D

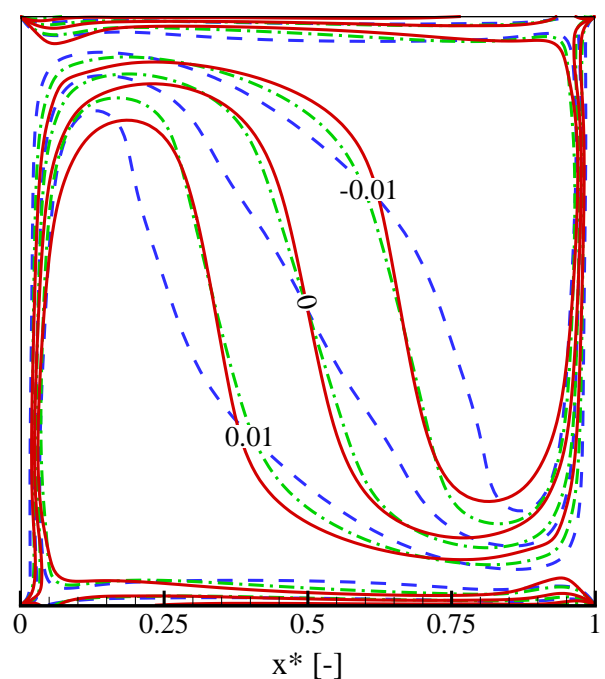

Figure 13: Dimensionless divergence of the radiative flux $-\nabla \cdot \mathbf{q}_{\mathrm{r}}^{*}$ in the plane $y^{*}=0.5$ for case B and $\mathrm{D}$ at $R a=10^{6}$ and $L=1 m$ (dashed line), $L=2 m$ (dash-dot line) and $L=3 m$ (solid line) 

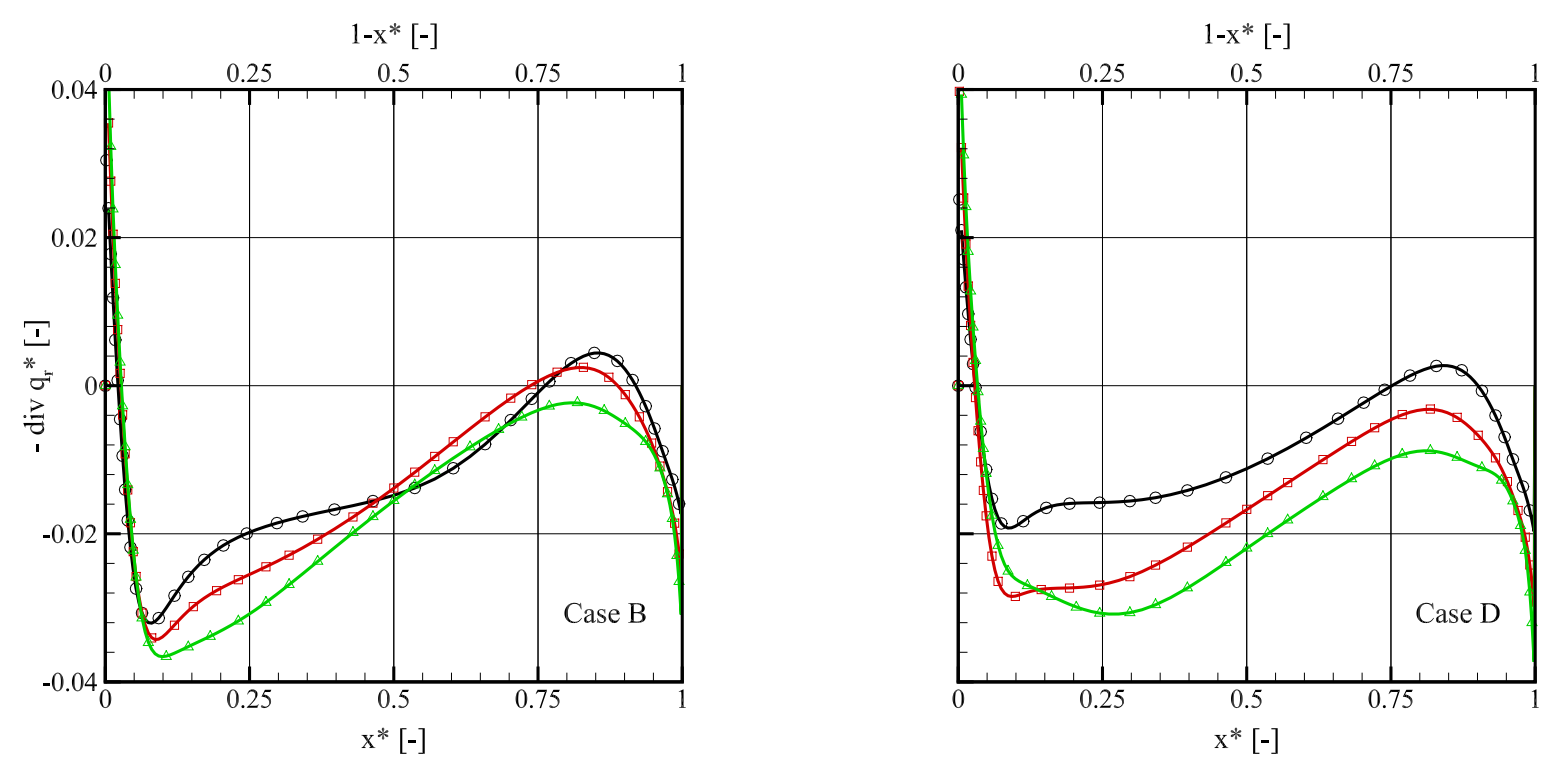

Figure 14: $-\boldsymbol{\nabla} \cdot \mathbf{q}_{\mathbf{r}}^{*}=f\left(x^{*}\right)$ at $y^{*}=z^{*}=0.9$ (symbols) compared to $-\boldsymbol{\nabla} \cdot \mathbf{q}_{\mathbf{r}}^{*}=-f\left(1-x^{*}\right)$ at $y^{*}=z^{*}=0.1$ (lines) in case $\mathrm{B}$ (left) and $\mathrm{D}$ (right) : $\mathrm{L}=1 \mathrm{~m}(\circ), \mathrm{L}=2 \mathrm{~m}(\square)$ and $\mathrm{L}=3 \mathrm{~m}(\triangle)$. 

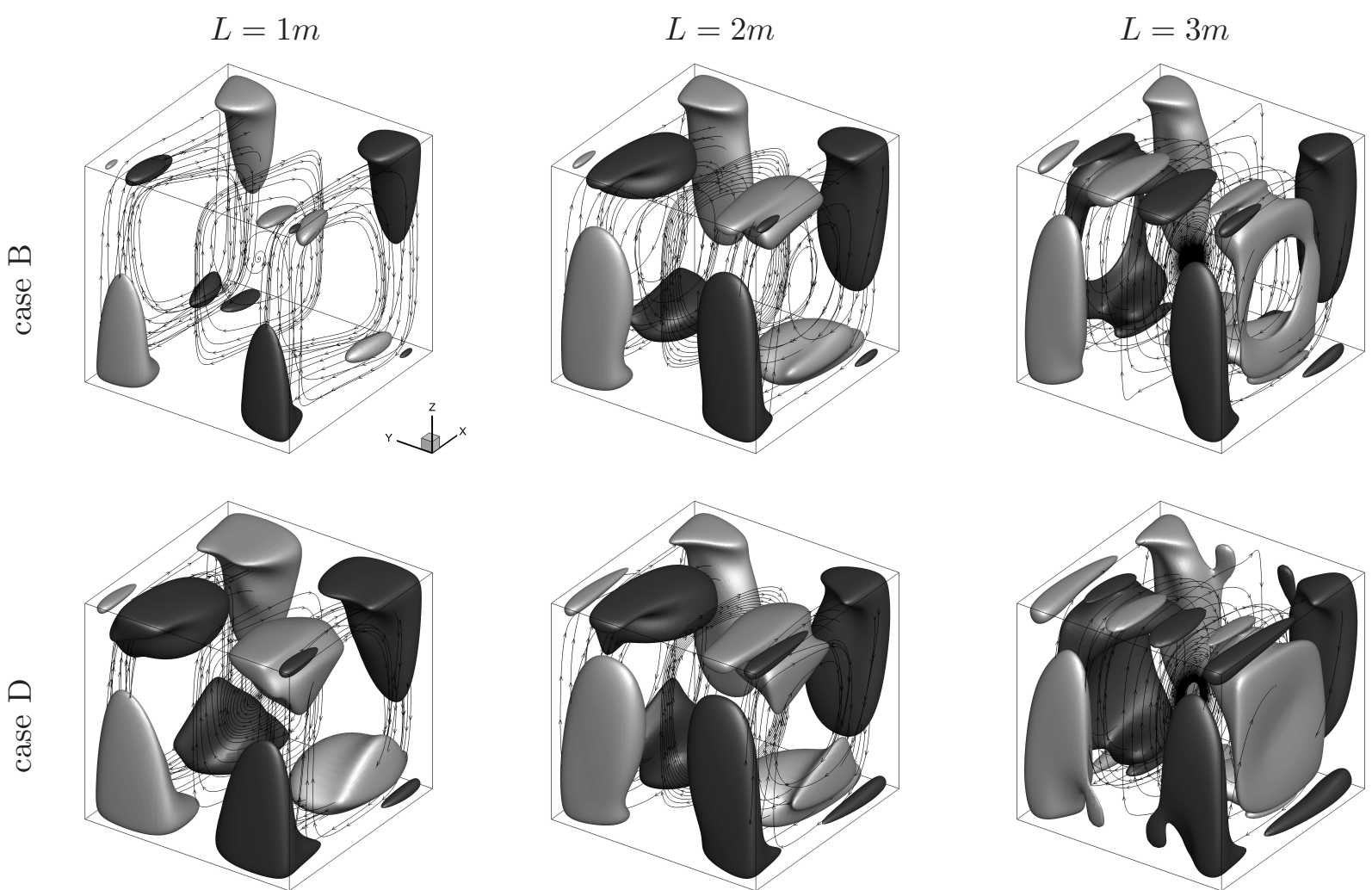

Figure 15: Isosurfaces of the dimensionless transverse component of the velocity $v^{*}$ at $R a=10^{6}: v^{*}=-0.02$ (dark grey) and $v^{*}=0.02$ (clear grey). 


\section{Tables}

\begin{tabular}{ccccc}
\hline Case & $\mathrm{A}$ & $\mathrm{B}$ & $\mathrm{C}$ & $\mathrm{D}$ \\
\hline Isothermal walls & $\varepsilon=1$ & $\varepsilon=1$ & $\varepsilon=1$ & $\varepsilon=1$ \\
Adiabatic walls & $\varepsilon=0$ & $\varepsilon=0$ & $\varepsilon=1$ & $\varepsilon=1$ \\
Gas nature & transparent & participating & transparent & participating
\end{tabular}

Table 1: Radiative boundary conditions and radiative properties of the medium corresponding to the four cases. 


\begin{tabular}{ccccccc}
\hline & & $\mathrm{A}$ & & & $\mathrm{B}$ & \\
& ref.* $^{*}$ & p.w.** & $\% \mathrm{E}$ & ref.* & p.w.** & $\% \mathrm{E}$ \\
\hline$N u^{c}$ & 8.64 & 8.65 & $<1 \%$ & 7.55 & 7.42 & $2 \%$ \\
$N u^{r}$ & 139.7 & 125.6 & $10 \%$ & 120.6 & 121.3 & $<1 \%$ \\
$S$ & 0.91 & 0.92 & $<1 \%$ & 0.35 & 0.36 & $2 \%$ \\
$\theta_{\text {top }}$ & 0.323 & 0.324 & $<1 \%$ & 0.207 & 0.206 & $<1 \%$ \\
$t_{\text {cpu }}$ & $20 \mathrm{~h}$ & $30 \mathrm{~h}$ & - & $170 \mathrm{~h}$ & $25 \mathrm{~h}$ & - \\
$N_{\text {proc }}$ & 4 & 1 & - & 94 & 1 & - \\
& & & & & & \\
\hline & & $\mathrm{C}$ & & & $\mathrm{D}$ & \\
& ref.* & p.w.** & $\% \mathrm{E}$ & ref.* & p.w.** & $\% \mathrm{E}$ \\
\hline$N u^{c}$ & 8.47 & 8.10 & $4 \%$ & 8.48 & 8.01 & $5 \%$ \\
$N u^{r}$ & 124.5 & 124.8 & $<1 \%$ & 119.6 & 119.9 & $<1 \%$ \\
$S$ & 0.42 & 0.41 & $4 \%$ & 0.26 & 0.24 & $8 \%$ \\
$\theta_{\text {top }}$ & 0.012 & 0.012 & $<1 \%$ & 0.016 & 0.016 & $<1 \%$ \\
$t_{\text {cpu }}$ & $20 \mathrm{~h}$ & $35 \mathrm{~h}$ & - & $100 \mathrm{~h}$ & $50 \mathrm{~h}$ & - \\
$N_{\text {proc }}$ & 8 & 1 & - & 94 & 1 & -
\end{tabular}

ref.*: [24], p.w.**: present work

Table 2: Convective and radiative Nusselt numbers, Stratification parameter and average temperature at the top wall for $R a=10^{6}$. Relative differences with values proposed by Soucasse et al. [24] and CPU time. 


\begin{tabular}{|c|c|c|c|c|c|c|}
\hline & \multicolumn{3}{|c|}{ A } & \multicolumn{3}{|c|}{ B } \\
\hline$L(m)$ & 1 & 2 & 3 & 1 & 2 & 3 \\
\hline$u_{\max }^{*}$ & 0.127 & 0.127 & 0.127 & 0.215 & 0.290 & 0.371 \\
\hline$v_{\max }^{*}$ & 0.026 & 0.026 & 0.026 & 0.051 & 0.079 & 0.090 \\
\hline$w_{\max }^{*}$ & 0.237 & 0.237 & 0.237 & 0.285 & 0.329 & 0.399 \\
\hline & \multicolumn{3}{|c|}{$\mathrm{C}$} & \multicolumn{3}{|c|}{$\mathrm{D}$} \\
\hline$\overline{L(m)}$ & 1 & 2 & 3 & 1 & 2 & 3 \\
\hline$u_{\max }^{*}$ & 0.217 & 0.218 & 0.219 & 0.251 & 0.329 & 0.406 \\
\hline$v_{\max }^{*}$ & 0.050 & 0.051 & 0.052 & 0.091 & 0.074 & 0.085 \\
\hline$w_{\max }^{*}$ & 0.279 & 0.280 & 0.280 & 0.323 & 0.353 & 0.420 \\
\hline
\end{tabular}

Table 3: Maximum velocities along $x, y$ and $z$ components for $R a=10^{6}$ 


\begin{tabular}{ccccccc}
\hline & & $\mathrm{A}$ & & & $\mathrm{B}$ & \\
\hline$L(m)$ & 1 & 2 & 3 & 1 & 2 & 3 \\
$S$ & 0.91 & 0.91 & 0.91 & 0.41 & 0.31 & 0.32 \\
$\theta_{\text {top }}$ & 0.32 & 0.32 & 0.32 & 0.22 & 0.17 & 0.14 \\
& & & & & & \\
\hline & & $\mathrm{C}$ & & & $\mathrm{D}$ & \\
\hline$L(m)$ & 1 & 2 & 3 & 1 & 2 & 3 \\
$S$ & 0.40 & 0.39 & 0.39 & 0.22 & 0.22 & 0.25 \\
$\theta_{\text {top }}$ & 0.01 & 0.01 & 0.01 & 0.02 & 0.01 & 0.01
\end{tabular}

Table 4: Stratification parameter and average temperature at the top wall for $R a=10^{6}$. 


\begin{tabular}{ccccccc}
\hline & \multicolumn{3}{c}{$\mathrm{A}$} & & & $\mathrm{B}$ \\
\hline$L(m)$ & 1 & 2 & 3 & 1 & 2 & 3 \\
$N u^{c}$ & 8.65 & 8.65 & 8.64 & 7.54 & 7.31 & 7.43 \\
$N u^{r}$ & 119.9 & 239.5 & 359.2 & 117.1 & 230.2 & 340.8 \\
$h_{r}$ & 3.09 & 3.09 & 3.08 & 3.01 & 2.96 & 2.93 \\
& & & & & & \\
\hline & & $\mathrm{C}$ & & & $\mathrm{D}$ & \\
\hline$L(m)$ & 1 & 2 & 3 & 1 & 2 & 3 \\
$N u^{c}$ & 8.08 & 8.38 & 8.54 & 8.02 & 8.28 & 8.68 \\
$N u^{r}$ & 119.2 & 238.5 & 358.1 & 115.9 & 228.6 & 338.9 \\
$h_{r}$ & 3.07 & 3.07 & 3.07 & 2.99 & 2.94 & 2.91
\end{tabular}

Table 5: Convective and radiative Nusselt numbers on hot wall $\left(x^{*}=0\right)$, and radiative heat transfer coefficient for $R a=10^{6}$ 